\title{
CLASSIFYING SPACES AND DIRAC OPERATORS COUPLED TO INSTANTONS
}

\author{
MARC SANDERS
}

\begin{abstract}
Let $M(k, S U(l))$ denote the moduli space of based gauge equivalence classes of $S U(l)$ instantons on principal bundles over $S^{4}$ with second Chern class equal to $k$. In this paper we use Dirac operators coupled to such connections to study the topology of these moduli spaces as $l$ increases relative to $k$. This "coupling" procedure produces maps $\partial_{u}: M(k, S U(l)) \rightarrow B U(k)$, and we prove that in the limit over $l$ such maps recover Kirwan's $[\mathrm{K}]$ homotopy equivalence $M(k, S U) \simeq B U(k)$. We also compute, for any $k$ and $l$, the image of the homology map $\left(\partial_{u}\right)_{*}: H_{*}(M(k, S U(l)) ; Z) \rightarrow H_{*}(B U(k) ; Z)$. Finally, we prove all the analogous results for $S p(l)$ instantons.
\end{abstract}

\section{INTRODUCTION}

Let $M(k, S U(l))$ be the moduli space of based gauge equivalence classes of $S U(l)$ instantons on principal bundles over $S^{4}$ with second Chern class (or charge) equal to $k$. Recently there has been much interest in how the topology of these spaces change as $k$ increases relative to $l$ (see, e.g., [BHMM], $[\mathrm{K}]$, and [Ti]). In this paper, in contrast, we shall use Dirac operators coupled to connections to study the topological behavior of these spaces as $l$ increases relative to $k$. The "coupling" of a Dirac operator to a connection associates to each element of $M(k, S U(l))$ an operator acting on the space of sections of a certain vector bundle. Associated to each self-dual connection is the vector space of sections in the kernel of its associated operator. This procedure defines a bundle, classified by a map which we shall refer to as the Dirac map, $\partial_{u}: M(k, S U(l)) \rightarrow B U(k)$. The topological properties of this map were first studied by Atiyah and Jones [AJ]. There are natural maps $M(k, S U(l)) \hookrightarrow M(k, S U(l+1))$, and it makes sense to define the Dirac map $\partial_{u}$ on the direct limit $M(k, S U)$. In this paper we show that this limit Dirac map, denoted $\partial_{u, \infty}$, realizes Kirwan's [K] homotopy equivalence between $M(k, S U)$ and $B U(k)$. Then we compute, for any $k$ and $l$, the image of the map $\left(\partial_{u}\right)_{*}: H_{*}(M(k, S U(l)) ; Z) \rightarrow H_{*}(B U(k) ; Z)$, where $\left(\partial_{u}\right)_{*}$ is the

Received by the editors November 1, 1994.

1991 Mathematics Subject Classification. Primary 53C07, 55P38; Secondary 55R45.

Key words and phrases. Instantons, Dirac operators, classifying space.

This work constitutes the author's Ph.D. dissertation, completed at Stanford University under the supervision of Professor Ralph Cohen, and was partially supported by an Alfred P. Sloan Foundation Fellowship. 
induced map in homology. Finally, we obtain similar results and do analogous computations for $S p(l)$ instantons.

By exploiting Donaldson's [D] monad description of $M(k, S U(l))$, Kirwan [K] used an algebro-geometric argument to show that $M(k, S U)$ and $B U(k)$ were homotopy equivalent. In the first part of this paper we use Donaldson's monad description to obtain a simpler, more direct, homotopy-theoretic proof that $M(k, S U) \simeq B U(k)$. In short, we show that $M(k, S U)$ is the quotient of a contractible space by a free $U(k)$ action. Similarly, for symplectic connections we show that $M(k, S p)$ is the quotient of a contractible space by a free $O(k)$ action. Hence, $M(k, S p) \simeq B O(k)$.

In the second part of this paper we define the Dirac maps

$$
\partial_{u}: M(k, S U(l)) \rightarrow B U(k)
$$

and

$$
\partial_{S p}: M(k, S p(l)) \rightarrow B O(k),
$$

describe the homotopy types of the spaces of charge-one instantons, and show that the limit maps $\partial_{u, \infty}: M(1, S U) \rightarrow B U(1)$ and $\partial_{S p, \infty}: M(1, S p) \rightarrow$ $B O(1)$ are homotopy equivalences. We then prove that Taubes' gluing maps, which give an associative $\mathrm{H}$-space structure to the space of instantons [T1], are compatible via the Dirac maps with the Whitney sum operation on line bundles. This compatibility in conjunction with the previous results for charge-one instantons allows us to conclude that the limit Dirac map $\partial_{u, \infty}: M(k, S U) \rightarrow$ $B U(k)$ is a homology equivalence, and therefore a homotopy equivalence, for $k>1$. After a more careful analysis of $\partial_{S p, \infty}$ on the level of universal covers, we show that it is also a homotopy equivalence for all $k$.

Finally in part three, we compute the images of the compositions

$$
\left(\partial_{u}\right)_{*}: H_{*}(M(k, S U(l)) ; Z) \rightarrow H_{*}(B U(k) ; Z)
$$

and

$$
\left(\partial_{S p}\right)_{*}: H_{*}\left(M(k, S p(l)) ; Z_{2}\right) \rightarrow H_{*}\left(B O(k) ; Z_{2}\right) .
$$

In the spirit of the splitting principle of bundle theory, we show that it suffices to calculate the image of $(\partial)_{*}$ when restricted to the space of "glued" charge-one instantons, and we make these calculations by exploiting earlier results on charge-one instantons and the relationship between Taubes' gluing and the Whitney sum operation. Combining this information with results on the rational homotopy type of $\Omega^{3} S U(l)$ enables us to conclude that the image, $\operatorname{Im}\left(\left(\partial_{\mathcal{u}}\right)_{*}\right) \subset H_{*}(B U ; Z)$, is the intersection of the images of the two maps

$$
H_{*}(B U(k) ; Z)
$$

$$
\begin{gathered}
\downarrow \\
H_{*}\left(\Omega_{k}^{3} S U(l) ; Z\right) \quad \longrightarrow \quad H_{*}(B U ; Z),
\end{gathered}
$$

where $H_{*}\left(\Omega_{k}^{3} S U(l) ; Z\right) \rightarrow H_{*}(B U ; Z)$ is induced by Bott periodicity. More precisely, let $H_{*}(Z \times B U ; Z) \cong Z\left[b_{0}, b_{0}^{-1}, b_{1}, b_{2}, \ldots\right]$ where $b_{i} \in$ 
$H_{2 i}(B U(1) ; Z)$ is a generator. Define

$$
\operatorname{length}\left(b_{j_{1}}^{i_{1}} \cdots b_{j_{r}}^{i_{r}}\right)=\sum_{s} i_{s} .
$$

It will be shown that $\operatorname{Im}\left(\left(\partial_{\mathcal{u}}\right)_{*}\right)$ is the set of all linear combinations of monomials in $b_{0}, b_{1}, \ldots, b_{l-2}$ which have length equal to $k$.

A more delicate argument, using results of Boyer and Mann [BM] on the compatibility up to homotopy of Taubes gluing with the natural $C_{3}$-operad structure of $\Omega^{3} S p(l)$, as well as results from iterated loop space theory, gives the symplectic analogue of the above theorem: $\operatorname{Im}\left(\left(\partial_{S p}\right)_{*}\right)$, viewed as a subset of $H_{*}\left(B O ; Z_{2}\right)$, is the intersection of the images of the maps

$$
\begin{aligned}
& H_{*}\left(B O(k) ; Z_{2}\right) \\
& \downarrow \\
& H_{*}\left(\Omega_{k}^{3} S p(l) ; Z_{2}\right) \quad \longrightarrow \quad H_{*}\left(B O ; Z_{2}\right)
\end{aligned}
$$

where the bottom map again is induced by the Bott periodicity map $\Omega_{k}^{3} S p(l) \rightarrow$ $\Omega_{k}^{3} S p \simeq B O$.

An immediate corollary of the above results is that the index bundle of the Dirac operator coupled to instantons has no nowhere-zero sections. Were such a bundle to have a section, the Dirac map $\partial_{u}$ would factor through $B U^{\prime}(k-1)$. This would imply that the $\operatorname{Im}\left(\left(\partial_{u}\right)_{*}\right)$ could contain no monomials in $b_{i}$ of length $k$, contradicting our theorem.

In a future paper, we will consider $S O(l)$ instantons. In particular we will show that there is a homotopy equivalence $M(k, S O) \simeq B S p(k)$ and that this equivalence can be realized by a Dirac-type map.

\section{2. $M(k, S U)$ AND $B U(k)$}

The ADHM construction [ADHM] identifies the space of instantons with certain holomorphic bundles over complex projective space, and Donaldson used a monad construction to characterize such bundles in terms of a quotient of a set of sequences of complex matrices by a natural action of $U(k)$. More precisely, we have the theorem of Donaldson:

Proposition 1 (Donaldson [D]). Let $A(k, S U(l))$ be the space of four-tuples of complex matrices $\left(\alpha_{1}, \alpha_{2}, a, b\right)$, where $\alpha_{i}$ is $k \times k, a$ is $l \times k$ and $b$ is $k \times l$, satisfying:

(i) $\left[\alpha_{1}, \alpha_{2}\right]+b a=0$

(ii) $\left[\alpha_{1}, \alpha_{1}^{*}\right]+\left[\alpha_{2}, \alpha_{2}^{*}\right]+b b^{*}-a^{*} a=0$

(iii) For any $\lambda, \mu \in C$ :

$$
\left(\begin{array}{c}
\alpha_{1}+\lambda \\
\alpha_{2}+\mu \\
a
\end{array}\right)
$$

is injective, and $\left(\lambda-\alpha_{1}, \alpha_{2}-\mu, b\right)$ is of maximal rank. 
Then there is a natural action of $U(k)$ on $A(k, S U(l))$ given by:

$$
p \cdot\left(\alpha_{1}, \alpha_{2}, a, b\right)=\left(p \alpha_{1} p^{-1}, p \alpha_{2} p^{-1}, a p^{-1}, p b\right)
$$

for $p \in U(k)$, and $M(k, S U(l))$ is homeomorphic to $A(k, S U(l)) / U(k)$.

Although it has been noted in the literature that the action of $U(k)$ on the spaces $A(k, S U(l))$ is a free action (see, e.g., $[\mathrm{K}]$ ), a proof of this fact has not to our knowledge been written down in the literature. For the sake of completeness, we give such a proof here.

Lemma 2. The natural action of $U(k)$ on the space $A(k, S U(l))$ is free.

Proof. Let $x=\left(\alpha_{1}, \alpha_{2}, a, b\right) \in A(k, S U(l))$ and let $d \in U(k)$ be such that $d \cdot x=x$. Without loss of generality we may assume that $d$ is the diagonal matrix $\left(z_{1}, \ldots, z_{k}\right)$ where for some $j>0 \quad z_{1}, \ldots, z_{j} \neq 1$ and $z_{j+1}=\ldots=$ $z_{k}=1$. (If $d$ is not diagonal then there is some $p \in U(k)$ such that $p d p^{-1}$ is diagonal and fixes $p x$.) Now since $z_{1}$ through $z_{j}$ are not 1 and $a d^{-1}=a$, we must have the first $j$ columns of $a$ equal to zero. Similarly, the first $j$ rows of $b$ must also be zero. This implies that

$$
b a=\left(\begin{array}{cc}
0_{j, j} & 0_{j, k-j} \\
0_{k-j, j} & *
\end{array}\right),
$$

where $0_{m, n}$ is the $m \times n$ zero matrix. Now consider the effect of conjugation by $d$ on $\alpha_{i} . d \alpha_{i} d^{-1}$ is obtained from $\alpha_{i}$ by multiplying row $r$ by $z_{r}$ and column $r$ by $z_{r}^{-1}$. So, if $d \cdot \alpha_{i}=\alpha_{i}$ then we can write

$$
\alpha_{i}=\left(\begin{array}{cc}
M_{i} & 0_{j, k-j} \\
0_{k-j, j} & *
\end{array}\right)
$$

for some $j \times j$ matrix $M_{i}$. Since $\left[\alpha_{1}, \alpha_{2}\right]+b a=0$, we must have $M_{1} M_{2}=$ $M_{2} M_{1}$. Let $v \in C^{j}$ be a common eigenvector for $M_{1}$ and $M_{2}$ and suppose $M_{1} v=\lambda v$ and $M_{2} v=\mu v$. Consider the matrix

$$
\left(\begin{array}{c}
\alpha_{1}-\lambda \\
\alpha_{2}-\mu \\
a
\end{array}\right)=\left(\begin{array}{cc}
M_{1}-\lambda & 0_{j, k-j} \\
0_{k-j, j} & *-\lambda \\
M_{2}-\mu & 0_{j, k-j} \\
0_{k-j, j} & *-\mu \\
0_{l, j} & a_{l, k-j}
\end{array}\right)
$$

where $a_{l, k-j}$ is the last $k-j$ columns of $a$. Any nonzero vector $(v, 0, \ldots$, 0 ), where there are $k-j$ zeros, is clearly in its nullspace. This contradicts the rank condition for the elements of $A(k, S U(l))$, and we conclude that the action is free. This completes the proof of the lemma.

We now construct the limit space $M(k, S U)$ and prove that it is homotopy equivalent to $B U(k)$. First notice that there is a $U(k)$ equivariant map from $A(k, S U(l)) \hookrightarrow A(k, S U(l+1))$ which sends each $\alpha_{i}$ to itself, sends $a$ to the $(l+1) \times k$ matrix made up of $a$ with an extra row of zeros on top, and sends $b$ to the $k \times(l+1)$ matrix made up of $b$ with an extra first column of zeros. On the level of monads, this adds to the bundle over $C P^{3}$ the trivial holomorphic 
line bundle. Thus, this map induces the natural inclusion $M(k, S U(l)) \hookrightarrow$ $M(k, S U(l+1))$ sending the connection $\omega$ to the connection $\omega \oplus d$, where $d$ is ordinary exterior differentiation. Notice that the charge $k$ is held fixed. Define $A(k, S U)$ to be the direct $\operatorname{limit}_{\lim _{l \rightarrow \infty}} A(k, S U(l))$ and $M(k, S U)$ to be the direct limit $\lim _{l \rightarrow \infty} M(k, S U(l))$. Note that there is a homeomorphism between $M(k, S U)$ and $A(k, S U) / U(k)$.

Theorem 3. $A(k, S U)$ is a contractible space with a free $U(k)$ action. Thus, $M(k, S U) \cong A(k, S U) / U(k) \simeq B U(k)$.

Proof. We need to show that $A(k, S U)$ is contractible. Since it is an algebraic subvariety and, therefore, homotopy equivalent to a C.W. complex, it will be sufficient to show that all of its homotopy groups are zero. To this end we prove that for any $k$ and $l$ there is an $r>l$ such that the map from $A(k, S U(l)) \rightarrow$ $A(k, S U(r))$ is homotopically trivial.

Consider the homotopy $H_{t}: A(k, S U(l)) \rightarrow A(k, S U(2 k+l))$ defined as follows:

$$
H_{t}\left(\left(\alpha_{1}, \alpha_{2}, a, b\right)\right)=\left((1-t) \alpha_{1},(1-t) \alpha_{2}, a_{t}, b_{t}\right)
$$

where

$$
a_{t}=\left(\begin{array}{c}
t I_{k} \\
0_{k, k} \\
(1-t) a
\end{array}\right) \text { and } b_{t}=\left(0_{k, k}, t I_{k},(1-t) b\right)
$$

and $I_{k}$ is the $k \times k$ identity matrix. To see that $H_{t}(x) \in A(k, S U(2 k+l))$ for any $x \in A(k, S U(l))$, we check that the three conditions are satisfied for all $0 \leq t \leq 1$. Condition (i) holds because $b_{t} a_{t}=(1-t)^{2} b a$, and for any matrices $A$ and $B:[(1-t) A,(1-t) B]=(1-t)^{2}[A, B]$. For condition (ii) notice that $b_{t} b_{t}^{*}=(1-t)^{2} b b^{*}+t^{2} I_{k}$ and similarly for $a_{t}^{*} a_{t}$. Thus, $b_{t} b_{t}^{*}-a_{t}^{*} a_{t}=$ $(1-t)^{2}\left(b b^{*}-a^{*} a\right)$, and condition (ii) holds. Now the rank condition obviously holds for all $t \neq 0$ because there is a full rank $k \times k$ block, $t I_{k}$, in both $a_{t}$ and $b_{t}$. For $t=0, H_{t}=H_{0}$ is just the inclusion $A(k, S U(l)) \hookrightarrow A(k, S U(2 k+l))$, so the rank condition is satisfied when $t=0$. Finally, note that $H_{1}$ is a constant map. This finishes the theorem.

\section{SyMPLECTIC InSTANTONS}

We begin with Donaldson's matrix description of symplectic instantons. Let $J$ be the standard skew form on $C^{2 l}$,

$$
J=\left(\begin{array}{cc}
0_{l, l} & I_{l} \\
-I_{l} & 0_{l, l}
\end{array}\right)
$$

and let $A(k, S p(l)) \subset A(k, S U(2 l))$ be those $\left(\alpha_{1}, \alpha_{2}, a, b\right)$ such that the $\alpha_{i}$ are symmetric and $a=J \cdot b^{t}$. The group $O(k)$ (viewed as a subset of $U(k)$ ) acts on $A(k, S p(l))$.

Proposition 4 (Donaldson [DK, Chapter 3.4]).

$$
M(k, S p(l)) \cong A(k, S p(l)) / O(k) .
$$

The remaining theorems and arguments in this section are quite similar to those of the previous section. However, before defining the limit space $A(k, S p)$, it is convenient to alter slightly our description of $M(k, S p(l))$ 
by constructing a space $A^{\prime}(k, S p(l))$, which is equivariantly homeomorphic to $A(k, S p(l))$. Essentially, this change amounts to choosing a different skew form on $C^{2 l}$. Let $J^{\prime}$ be the $2 l \times 2 l$ complex $2 \times 2$ block diagonal matrix

$$
J^{\prime}=\operatorname{Diag}(\underbrace{J_{2}, \ldots, J_{2}}_{l}) \text { where } J_{2}=\left(\begin{array}{cc}
0 & 1 \\
-1 & 0
\end{array}\right)
$$

Notice that there exists a $\sigma \in O(2 l), \sigma J^{\prime} \sigma^{-1}=J$. Let $A^{\prime}(k, S p(l))$ be the subset of $A(k, 2 l)$ where the $\alpha_{i}$ are symmetric and $a=J^{\prime} b^{t}$. We can define an equivariant homeomorphism from $A(k, S p(l))$ to $A^{\prime}(k, S p(l))$ by mapping $\left(\alpha_{1}, \alpha_{2}, a, b\right)$ to $\left(\alpha_{1}, \alpha_{2}, \sigma^{-1} a, b \sigma\right)$. Since $\sigma \in O(2 l)$ and, therefore, $\sigma^{*}=$ $\sigma^{-1}=\sigma^{t}$, conditions (i), (ii), and (iii) are satisfied by $\left(\alpha_{1}, \alpha_{2}, \sigma^{-1} a, b \sigma\right)$. Also, since $a=J b^{t}$, we have $\sigma^{-1} a=\sigma^{-1} J b^{t}=J^{\prime} \sigma^{-1} b^{t}=J^{\prime}(b \sigma)^{t}$. Thus, the map from $A(k, S p(l))$ to $A^{\prime}(k, S p(l))$ is well-defined, and it is invertible because $\sigma$ is. (It is equivariant because $\sigma$ acts on the opposite side of the $O(k)$ action.) Thus, $M(k, S p(l)) \cong A^{\prime}(k, S p(l)) / O(k)$. Now there are natural $O(k)$ equivariant maps $A^{\prime}(k, S p(l)) \hookrightarrow A^{\prime}(k, S p(l+1))$ which send each $\alpha_{i}$ to itself, send $a$ to the $(2 l+2) \times k$ matrix made up of $a$ with an extra 2 rows of zeros on top, and send $b$ to the $k \times(2 l+2)$ matrix made up of $b$ with an extra 2 first columns of zeros. As in the unitary case, we can define $A^{\prime}(k, S p)$ to be the direct limit of the spaces $A^{\prime}(k, S p(l))$ and, $M(k, S p)$ to be $A^{\prime}(k, S p) / O(k)$.

Theorem 5. $M(k, S p) \simeq B O(k)$.

Proof. The action of $O(k)$ on $A(k, S p(l))$ is free because it is a restriction of the action of $U(k)$ on $A(k, S U(2 l))$. This implies that the action on $A^{\prime}(k, S p(l))$ is also free. Thus, it is enough to show that $A^{\prime}(k, S p)$ is contractible. As in the unitary case (Theorem 3), define $H_{t}: A^{\prime}(k, S p(l)) \rightarrow$ $A^{\prime}(k, S p(k+l))$ as follows:

$$
H_{t}\left(\left(\alpha_{1}, \alpha_{2}, a, b\right)=\left((1-t) \alpha_{1},(1-t) \alpha_{2}, a_{t}, b_{t}\right)\right)
$$

where

$$
a_{t}=\left(\begin{array}{c}
t \cdot(I 0)_{k} \\
(1-t) a
\end{array}\right) \text { and } b_{t}=\left(t \cdot(0 I)_{k},(1-t) b\right) \text {. }
$$

Here $(I 0)_{k}$ is the $2 k \times k$ matrix where, for $1 \leq i \leq k$, row $2 i$ is zero and row $2 i-1$ is the $i$ th row of the $k \times k$ identity matrix. $(0 I)_{k}$ is obtained by transposing $(I 0)_{k}$ and then switching column $2 i$ with column $2 i-1$. For example:

$$
(I 0)_{2}=\left(\begin{array}{ll}
1 & 0 \\
0 & 0 \\
0 & 1 \\
0 & 0
\end{array}\right) \text { and }(0 I)_{2}=\left(\begin{array}{cccc}
0 & 1 & 0 & 0 \\
0 & 0 & 0 & 1
\end{array}\right)
$$

Just as in the unitary case, any element in the image of $H_{t}$ will satisfy conditions (i), (ii), and (iii) for all $0 \leq t \leq 1$. Furthermore, $(1-t) \alpha_{i}$ is symmetric if $\alpha_{i}$ is. Finally, one can check directly that $a_{t}$ and $b_{t}$ were chosen so that if $a=J^{\prime} b^{t}$, then for any $t, a_{t}=J^{\prime} b_{t}^{t}$. So $H_{t}$ is well-defined. Now, $H_{0}$ is just the natural inclusion, and $H_{1}$ is a constant map. As in the unitary case, this implies that $A^{\prime}(k, S p)$ is contractible, and the proof of the theorem is complete. 


\section{Charge-one instantons}

Before studying Dirac operators and using them to define homotopy equivalences $M(k, S U) \simeq B U(k)$ and $M(k, S p) \simeq B O(k)$, we need to examine more closely instantons on bundles with $c_{2}=1$. Boyer, Mann and Waggoner [BMW] showed that $M(1, S U(l))$ and $M(1, S p(l))$ are homotopy equivalent to the quotient of the respective Lie group by the subgroup consisting of elements in the centralizer of $S U(2)=S p(1)$. By exploiting Donaldson's monad description of instantons, we are able to give an alternate description and proof of these results.

\section{Lemma 6.}

(i) $M(1, S U(l))$ is homotopy equivalent to the unit tangent bundle of $C P^{l-1}$.

(ii) $M(1, S p(l)) \simeq R P^{4 l-1}$.

Proof. From Proposition 1 we have $M(1, S U(l)) \cong A(1, S U(l)) / U(1)$. Examining the relations which must hold on a four-tuple $\left(\alpha_{1}, \alpha_{2}, a, b\right) \in$ $A(1, S U(l))$ and noticing that the $U(1)$ action on $\alpha_{i}$ is trivial, we see that $M(1, S U(l))$ is homeomorphic to

$$
C^{2} \times\left\{\left.\left(z_{1}, \ldots, z_{l} ; w_{1}, \ldots, w_{l}\right)\left|\vec{z}, \vec{w} \in C^{l} \backslash\{0\}, \sum_{i=1}^{n} z_{i} w_{i}=0, \sum_{i=1}^{n}\right| z_{i}\right|^{2}=\sum_{i=1}^{n}\left|w_{i}\right|^{2}\right\} / S^{1} .
$$

Thus, if we define

$$
M^{\prime}(1, S U(l)) \equiv\left\{(\vec{z} ; \vec{w})\left|\vec{z}, \vec{w} \in C^{l} \backslash\{0\}, \sum z_{i} w_{i}=0,\right| z|=| w \mid=1\right\} / S^{1}
$$

then $M^{\prime}(1, S U(l)) \simeq M(1, S U(l))$. There is an obvious map

$$
M^{\prime}(1, S U(l)) \stackrel{\pi}{\longrightarrow} C P^{l-1}
$$

where $\pi(\vec{z} ; \vec{w})=[\vec{z}] \in C P^{l-1}$. Notice that if $[\vec{a}]=\left[\left(a_{1}, \ldots, a_{l}\right)\right] \in C P^{l-1}$ then

$$
\pi^{-1}[\vec{a}] \cong\left\{\left.\left(w_{1}, \ldots, w_{l}\right)\left|\sum\right| w_{i}\right|^{2}=1 \text { and } \sum a_{i} w_{i}=0\right\} \cong S^{2 l-3}
$$

We leave to the reader the verification that $\pi: M^{\prime}(1, S U(l)) \longrightarrow C P^{l-1}$ is a locally trivial $S^{2 l-3}$ fibration. In fact, let $\langle$,$\rangle denote the standard hermitian$ inner product on $C^{l}$. Let $N_{l}$ be the set

$$
N_{l}=\left\{(\vec{z} ; \vec{w})\left|\vec{z}, \vec{w} \in C^{l} \backslash\{0\},\langle\vec{z}, \vec{w}\rangle=0,\right| z|=| w \mid=1\right\} / S^{1}
$$

where $\lambda \in S^{1}$ acts by $\lambda \cdot(\vec{z} ; \vec{w})=(\lambda \vec{z} ; \lambda \vec{w})$. Since $C P^{l-1}$ can be identified with the quotient $S^{2 l-1} / S^{1}, N_{l}$ is precisely the unit tangent bundle of $C P^{l-1}$. Moreover, the map $(\vec{z} ; \vec{w}) \mapsto(\vec{z} ; \vec{w})$ induces a homeomorphism from $M^{\prime}(1, S U(l))$ onto $N_{l}$. Hence, $M(1, S U(l))$ is homotopy equivalent to the unit tangent bundle of $C P^{l-1}$.

For part (ii) of the lemma, Proposition 4 implies that

$$
M(1, S p(l)) \cong A(1, S p(l)) / O(1) \simeq C^{2 l} \backslash\{0\} / Z_{2} \simeq R P^{4 l-1} .
$$

The following is an immediate consequence of the above lemma. 


\section{Corollary 7.}

(i) $H_{i}(M(1, S U(l)) ; Z) \cong H_{i}(B U(1) ; Z)$ for $i \leq 2 l-4$.

(ii) $H_{2 l-2}(M(1, S U(l)) ; Z)=0$ and $H_{2 l-3}(M(1, S U(l)) ; Z)=Z / l$.

(iii) $H_{i}\left(M(1, S p(l)) ; Z_{2}\right) \cong H_{i}\left(B O(1) ; Z_{2}\right)$ for $i \leq 4 l-1$.

\section{TAubes gluing and the Dirac maP}

Let $G$ denote either $S U(l)$ or $S p(l)$. Let $B(k, G)$ be the space of all gauge equivalence classes of connections on principal $G$-bundles, $P_{k}$, over $S^{4}$ with $c_{2}\left(P_{k}\right)=k$. This space is known to be homotopy equivalent to $\Omega_{k}^{3} G$, and Atiyah and Jones [AJ] exhibit a particular map $\Theta_{k}^{G}: B(k, G) \rightarrow \Omega_{k}^{3} G$ which realizes this equivalence. Let $i_{k, l}^{G}: M(k, G) \rightarrow \Omega_{k}^{3} G$ denote the inclusion of equivalence classes of self-dual connections into $B(k, G)$ followed by $\Theta_{k}^{G}$. When no confusion can occur, we will drop the superscript when writing $i_{k, l}^{G}$. We now recall the important gluing construction for instantons introduced by Taubes [T1]. Taubes shows that for a given $l$, the space $\coprod_{k>0} M(k, G)$ has an $\mathrm{H}$-space structure and that this structure is compatible up to homotopy with the group structure on $\Omega^{3} G$ [T2], viewed as the space of all based gauge equivalence classes of connections. In particular the following diagram commutes up to homotopy:

$$
\begin{array}{ccc}
M(1, G)^{k} & \longrightarrow & M(k, G) \\
\left(i_{1, l}\right)^{k} \downarrow & & \downarrow i_{k, l} \\
\left(\Omega_{1}^{3} G\right)^{k} & \longrightarrow & \Omega_{k}^{3} G
\end{array}
$$

where the horizontal maps give the multiplicative structures.

Next, we describe the construction of what we refer to as the Dirac map

$$
\partial_{u}: M(k, S U(l)) \rightarrow B U(k) .
$$

Given $P_{k}$, a principal $S U(l)$ bundle over $S^{4}$, let $\omega$ be a connection on the associated vector bundle $E_{k}$. Let $S$ be the canonical complex bundle of spinors on $S^{4}$ with its canonical connection $\nabla_{s}$. The tensor product bundle $S \otimes E_{k}$ inherits a Clifford module structure from the one on $S$, and we can view $\omega \otimes \nabla_{s}$ as a connection on this bundle. This connection gives rise to a Dirac operator

$$
D_{\omega}: \Gamma\left(S \otimes E_{k}\right) \rightarrow \Gamma\left(S \otimes E_{k}\right)
$$

where $\Gamma\left(S \otimes E_{k}\right)$ is the space of smooth sections of $S \otimes E_{k}$. Notice that the group of bundle automorphisms of $E_{k}$ acts on the space $\Gamma\left(S \otimes E_{k}\right)$. The spinor bundle naturally splits into positive and negative valued spinors, $S=S^{+} \oplus S^{-}$, and the Dirac operator interchanges the two summands. We shall be concerned with the operator

$$
D_{\omega}^{+}: \Gamma\left(S^{+} \otimes E_{k}\right) \rightarrow \Gamma\left(S^{-} \otimes E_{k}\right),
$$

which is known to be Fredholm, in an appropriate Sobolev completion, and of index $k[\mathrm{AJ}]$. Using Bochner type vanishing theorems, one can show that if $\omega$ is a self-dual connection then $\operatorname{Coker}\left(D_{\omega}^{+}\right)=0$. (See, for example, [AHS].) Therefore, the space of sections in the kernel of $D_{\omega}^{+}$gives a well-defined vector space associated to the connection $\omega$. There is an equivariance of this kernel under 
gauge transformation in the sense that if $\sigma \in \operatorname{Ker}\left(D_{\omega}\right)$ then $g \sigma \in \operatorname{Ker}\left(D_{g \omega}\right)$, for any $g$ in the gauge group. Passing to gauge equivalence classes, we obtain an isomorphism class of a vector bundle of rank $k$ over $M(k, S U(l))$. This bundle is classified by a map $\partial_{u}: M(k, S U(l)) \rightarrow B U(k)$. Atiyah and Jones showed that the map $\partial_{u}$ has a natural extension to the entire space of based gauge equivalence classes of connections as a map $\tilde{\partial_{u}}: B(k, S U(l)) \rightarrow\{k\} \times B U \subset Z \times$ $B U$ : each connection gives rise to a Fredholm operator of index $k$ and the space of such operators is homotopy equivalent to the $k$ th component of $Z \times$ $B U$.

A similar Dirac map exists for symplectic connections. However, because $S$ has a quaternionic structure, given by some complex anti-linear bundle automorphism $J_{s}$, where $J_{s} \circ J_{s}=-1$, and the symplectic vector bundle $E_{k}$ also has a quaternionic structure, $J_{E}$, the tensor product bundle has a real structure given by the tensor product $J_{S} \otimes J_{E}$. This is turn defines a real structure on the vector space of sections $\Gamma\left(S \otimes E_{k}\right)$. The real subspaces given by the real structure will be preserved by the Dirac operator, and the kernels of the Dirac operators then define a real vector bundle over $M(k, S p(l))$. Hence, $\partial_{S p}$ can be viewed as a map from $M(k, S p(l))$ to $B O(k)$. As before, we may also define $\tilde{\partial}_{S p}: \Omega_{k}^{3} S p(l) \rightarrow\{k\} \times B O \subset Z \times B O$, where $Z \times B O$ is identified with the space of real Fredholm operators. In short, there exists homotopy commutative diagrams

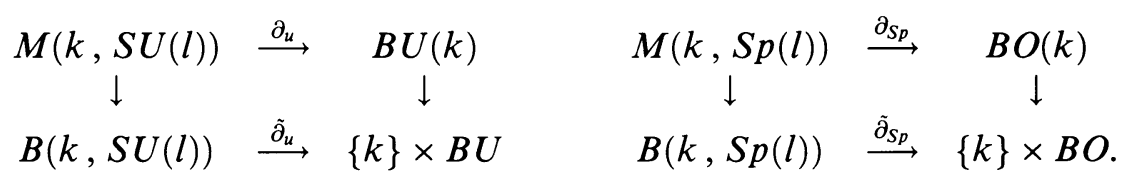

Atiyah and Jones ([AJ], Theorem 4.12) also observed that in the limit over $l$, $\tilde{\partial}_{u}$ realizes Bott periodicity. More precisely, we have the following proposition.

Proposition 8 [AJ]. There exists a homotopy commutative diagram

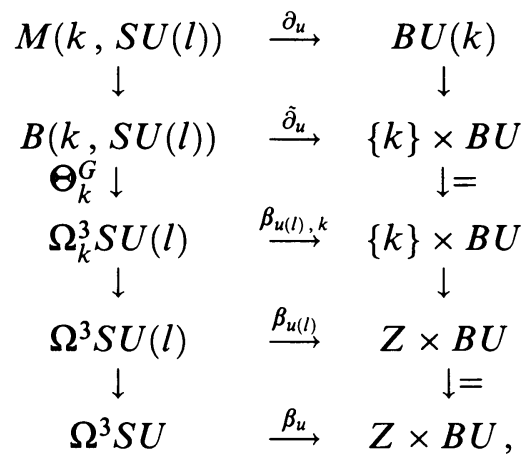

where $\beta_{u}$ is a homotopy equivalence preserving the natural $H$-space structures, and $\beta_{u(l)}$ and $\beta_{u(l), k}$ are its natural restrictions to the appropriate loop spaces.

In fact $\beta_{u}$ is obtained by two-fold looping the Bott equivalence $\Omega U \simeq Z \times$ $B U$, then identifying $\Omega^{2}(Z \times B U)$ with $\Omega^{2}(B U) \simeq \Omega U \simeq Z \times B U$ by applying the Bott equivalence again. Thus, $\beta_{u}$ is an infinite loop map. There also exists a diagram for symplectic connections analogous to the one above. In particular 
there are maps $\beta_{S p(l), k}, \beta_{S p(l)}$ and $\beta_{S p}$, where $\beta_{S p}$ realizes the equivalence $\Omega^{3} S p \simeq Z \times B O[\mathrm{AJ}]$.

For sake of completeness we now show that, as in the unitary case, $\beta_{S p}$ preserves the H-space structures, and is, in fact, an infinite loop map (cf. [AJ], Appendix, proof of Lemma 4.8). Let $E_{k}$ and $E_{j}$ be $S p(n)$ bundles over $S^{4}$ with second Chern classes $k$ and $j$ respectively. Let $L_{1}^{2}\left(E_{r}\right)$ and $L^{2}\left(E_{r}\right)$ be the Sobolev completions of the space of smooth sections $\Gamma\left(S^{+} \otimes E_{r}\right)$ in the given norm. Explicitly, a section $\sigma$ is in $L_{1}^{2}$ if both $\sigma$ and $\nabla \sigma$ have finite $L^{2}$ norm. Let $L_{1}^{2}\left(E_{r}\right)_{R}$ and $L^{2}\left(E_{r}\right)_{R}$ denote the Sobolev completions of the subspace of real sections $\Gamma\left(S^{+} \otimes E_{r}\right)_{R}$. Let $\mathscr{F}_{R}\left(\mathbf{H}_{1}, \mathbf{H}_{2}\right)$ denote the space of real Fredholm operators from the real Hilbert space $\mathbf{H}_{1}$ to the real Hilbert space $\mathbf{H}_{2}$. If we choose an isomorphism $S^{-} \cong S^{+}$, then the coupling of a symplectic connection to a Dirac operator defines a map

$$
B(k, S p(n)) \longrightarrow \mathscr{F}_{R}\left(L_{1}^{2}\left(E_{k}\right)_{R}, L^{2}\left(E_{k}\right)_{R}\right) \simeq Z \times B O .
$$

The direct sum of connections

$$
\left(E_{k}, \omega_{k}\right) \times\left(E_{j}, \omega_{j}\right) \mapsto\left(E_{k} \oplus E_{j}, \omega_{k} \oplus w_{j}\right)
$$

gives a map $B(k, S p(n)) \times B(j, S p(n)) \rightarrow B(k+j, S p(2 n))$ which then makes the following diagram commute:

$$
\begin{array}{ccc}
B(k, S p(n)) \times B(j, S p(n)) & \longrightarrow & \mathscr{F}_{R}\left(L_{1}^{2}\left(E_{k}\right)_{R}, L^{2}\left(E_{k}\right)_{R}\right) \times \mathscr{F}\left(L_{1}^{2}\left(E_{j}\right)_{R}, L^{2}\left(E_{j}\right)_{R}\right) \\
\oplus \downarrow & \downarrow \oplus \\
B(k+j, S p(2 n)) & \longrightarrow & \mathscr{F}_{R}\left(L_{1}^{2}\left(E_{k}\right)_{R} \oplus L_{1}^{2}\left(E_{j}\right)_{R}, L^{2}\left(E_{k}\right)_{R} \oplus L^{2}\left(E_{j}\right)_{R}\right),
\end{array}
$$

where the right vertical map is defined by taking the direct sum of the operators. Now the direct sum of operators can be identified in a standard way with composition of operators on some larger Hilbert space $\mathbf{H}$. For example, one could take $\mathbf{H}$ to be the sum $L_{1}^{2}\left(E_{k}\right)_{R} \oplus L_{1}^{2}\left(E_{j}\right)_{R} \oplus L^{2}\left(E_{k}\right)_{R} \oplus L^{2}\left(E_{j}\right)_{R}$. Since composition of operators corresponds to the Whitney sum operation on $Z \times B O$, and the homotopy inverse of the equivalence $\Theta_{k}: B(k, S p(n)) \stackrel{\cong}{\rightrightarrows} \Omega_{k}^{3} S p(n)$ respects direct sums, there is a homotopy commutative diagram

$$
\begin{aligned}
& \Omega_{k}^{3} S p(n) \times \Omega_{j}^{3} S p(n) \stackrel{\beta_{S p(n), k} \times \beta_{S p(n), j}}{\longrightarrow} Z \times B O \times Z \times B O \\
& \oplus \downarrow \quad \downarrow \oplus \\
& \Omega_{k+j}^{3} S p(2 n) \quad \stackrel{\beta_{S p(2 n), k+j}}{\longrightarrow} Z \times B O .
\end{aligned}
$$

Finally, the standard rotation homotopy shows that the left vertical map is homotopic to the composition

$$
\Omega_{k}^{3} S p(n) \times \Omega_{j}^{3} S p(n) \stackrel{l}{\longrightarrow} \Omega_{k+j}^{3} S p(n) \hookrightarrow \Omega_{k+j}^{3} S p(2 n)
$$


where $l$ is the usual loop sum. Therefore, the two compositions

$$
\begin{aligned}
& \Omega_{k}^{3} S p(n) \times \Omega_{j}^{3} S p(n) \stackrel{\beta_{S p(n), k} \times \beta_{S(n), j}}{\longrightarrow} Z \times B O \times Z \times B O \\
& l \downarrow \quad \downarrow \oplus \\
& \Omega_{k+j}^{3} S p(n) \stackrel{\beta_{S(n), k+j}}{\longrightarrow} \quad Z \times B O
\end{aligned}
$$

are homotopic. This proves that $\beta_{S p}: \Omega^{3} S p \rightarrow Z \times B O$ is an H-map. Since it is a homotopy equivalence [AJ] and an H-map, it must, by a result of Cohen and Jones [CJ], be an infinite loop map.

We now show that $\partial_{u}$ can be defined on the limit space $M(k, S U)$. First note that from Donaldson's matrix description of $M(k, S U(n))$ (see Chapter 3) we see that the natural inclusion $M(k, S U(n)) \hookrightarrow M(k, S U(n+1))$, $\left(\omega, E_{k}\right) \mapsto\left(\omega \oplus d, E_{k} \oplus C\right)$, embeds $M(k, S U(n))$ as a closed submanifold of $M(k, S U(n+1))$. Hence, by simultaneous triangulation [Mu] or from the existence of tubular neighborhoods, the inclusions $M(k, S U(n)) \hookrightarrow$ $M(k, S U(n+1))$ are cofibrations. It follows that the direct limit $M(k, S U)$ is homotopy equivalent to the homotopy direct limit, denoted $M(k, S U)_{h}$. Thus, it suffices to define $\partial_{u}$ on $M(k, S U)_{h}$. To this end let $\mathscr{G}(k, V)$ denote the space of all $k$-dimensional subspaces of the vector space $V$. Let $A(k, S U(n))$ denote the space of all instantons before passing to gauge equivalence classes. Given an $S U(n)$ bundle $E_{k}$ with $c_{2}\left(E_{k}\right)=k$, the kernel of the Dirac operator coupled to an instanton on $E_{k}$ defines a map

$$
A(k, S U(n)) \longrightarrow \mathscr{G}\left(k, \Gamma\left(S^{+} \otimes E\right)\right) .
$$

More precisely, the coupling of a connection to an operator defines a bundle map

$$
\begin{array}{ccc}
\Gamma\left(S^{+} \otimes E_{k}\right) & \Gamma\left(S^{-} \otimes E_{k}\right) \\
\downarrow & \downarrow \\
A(k, S U(n)) \times_{\mathbf{G}} \Gamma\left(S^{+} \otimes E_{k}\right) & \longrightarrow & A(k, S U(n)) \times_{\mathbf{G}} \Gamma\left(S^{-} \otimes E_{k}\right) \\
\downarrow & & \downarrow \\
M(k, S U(n)) & = & M(k, S U(n)),
\end{array}
$$

where $\mathbf{G}$ is the based gauge group. Let

$$
\kappa(k, n)=\left\{(\omega, \sigma) \mid \sigma \in \operatorname{ker}\left(D_{\omega}^{+}\right)\right\} / \mathbf{G}
$$

denote the kernel of this bundle map. In other words, $\kappa(k, n)$ is the bundle classified by the Dirac map $\partial_{u}: M(k, S U(n)) \rightarrow B U(k)$. Since the untwisted Dirac operator on $S^{4}$ has no kernel ( $S^{4}$ has no harmonic spinors), the natural 
inclusion of bundles

$$
\begin{array}{ccc}
\kappa(k, n) & \hookrightarrow & \kappa(k, n+1) \\
\downarrow & & \downarrow \\
M(k, S U(n)) & \hookrightarrow & M(k, S U(n+1))
\end{array}
$$

defined by $(\omega, \sigma) \mapsto(\omega \oplus d, \sigma \oplus 0)$ is an isomorphism on fibers. Thus the pullback of $\kappa(k, n+1))$ via the inclusion $M(k, S U(n)) \hookrightarrow M(k, S U(n+1))$ is isomorphic to $\kappa(k, n))$. Therefore, the diagram

$$
\begin{array}{ccc}
M(k, S U(n)) & \hookrightarrow & M(k, S U(n+1)) \\
\partial_{u} \downarrow & & \downarrow \partial_{u} \\
B U(k) & = & B U(k)
\end{array}
$$

commutes up to homotopy. This implies that there is a map $\partial_{u, \infty}: M(k, S U)_{h}$ $\rightarrow B U(k)$. Precomposing with the equivalence $M(k, S U) \simeq M(k, S U)_{h}$, we obtain a map

$$
\partial_{u, \infty}: M(k, S U) \rightarrow B U(k) .
$$

This map is not necessarily uniquely determined, but any two choices, when restricted to $M(k, S U(n))$ will classify the bundle $\kappa(k, n)$, and this is the only property of the limit map we will need. An analogous argument for symplectic instantons shows that there exists a map $\partial_{S p, \infty}: M(k, S p) \rightarrow B O(k)$ with similar properties.

Theorem 9. For all $k, \partial_{u, \infty}: M(k, S U) \rightarrow B U(k)$ and $\partial_{S p, \infty}: M(k, S p) \rightarrow$ $B O(k)$ are homotopy equivalences.

Our proof consists of three parts. First we prove that for $k=1, \partial_{u, \infty}$ and $\partial_{S p, \infty}$ are homotopy equivalences. Then we show by using this special case, that for any $k, \partial_{u, \infty}$ induces an isomorphism in homology (which implies that it is a homotopy equivalence) and that $\partial_{S p, \infty}$ induces an isomorphism in homology with $Z_{2}$ coefficients. Finally we prove that on the level of universal covers, $\partial_{S p, \infty}$ induces an isomorphism on homotopy groups. This will force $\partial_{S p, \infty}$ to be a homotopy equivalence.

Lemma 10. The maps $\partial_{u, \infty}: M(1, S U) \rightarrow B U(1)$ and $\partial_{S p, \infty}: M(1, S p) \rightarrow$ $B O(1)$ are homotopy equivalences.

Proof. Because $M(1, S U) \simeq B U(1) \simeq K(Z, 2)$ and $M(1, S p) \simeq B O(1) \simeq$ $K\left(Z_{2}, 1\right)$, it suffices to show that $\partial_{u, \infty}$ induces an isomorphism on the second homotopy group, $\pi_{2}$, and $\partial_{S p, \infty}$ induces an isomorphism on the fundamental group $\pi_{1}$. We have a homotopy commutative diagram

$$
\begin{aligned}
& M(1, S U(3)) \hookrightarrow M(1, S U) \stackrel{\partial_{u, \infty}}{\longrightarrow} B U(1) \\
& i_{1,3} \downarrow \\
& \Omega_{1}^{3} S U(3) \quad \hookrightarrow \quad \Omega_{1}^{3} S U \stackrel{\simeq}{\longrightarrow}\{1\} \times B U,
\end{aligned}
$$


and we know by a previous lemma that

$$
\pi_{2}\left(M ( 1 , S U ( 3 ) ) \cong H ^ { 2 } \left(M(1, S U(3)) \cong Z \cong \pi_{2}\left(\Omega_{1}^{3} S U(3)\right) .\right.\right.
$$

Furthermore, the inclusion $M(1, S U(3)) \hookrightarrow \Omega_{1}^{3} S U(3)$ induces an isomorphism on $\pi_{2}[\mathrm{~K}]$. Since $\Omega_{1}^{3} S U(3) \rightarrow \Omega_{1}^{3} S U \hookrightarrow\{1\} \times B U$ is an isomorphism on $\pi_{2}$, it follows by the diagram above that $\partial_{u, \infty}: M(1, S U) \rightarrow B U(1)$ must be an isomorphism on $\pi_{2}$ as well.

A similar discussion shows that $\partial_{S p, \infty}: M(1, S p) \rightarrow B O(1)$ is an equivalence: there is a homotopy commutative diagram

$$
\begin{array}{cccc}
M(1, S p(1)) & \hookrightarrow & M(1, S p) \stackrel{\partial_{S p, \infty}}{\longrightarrow} & B O(1) \\
i_{1, l} \downarrow & & & \downarrow \\
\Omega_{1}^{3} S p(1) & \hookrightarrow & \Omega_{1}^{3} S p \quad \stackrel{\simeq}{\longrightarrow}\{1\} \times B O,
\end{array}
$$

and the left vertical map is homotopic to the $J$-homomorphism from $S O(3)$ to $\Omega^{3} S^{3}$ [BM], and therefore, induces an isomorphism on $\pi_{1}$. Just as in the previous argument, this allows us to conclude that $\partial_{S p, \infty}: M(1, S p) \rightarrow B O(1)$ is also an isomorphism on $\pi_{1}$ and, therefore, an equivalence. This finishes the lemma.

Notice that from this lemma and Lemma 6, we can conclude that

$$
\left(\partial_{u}\right)_{*}: H_{*}(M(1, S U(n))) \rightarrow H_{*}(B U(1))
$$

is a surjection through dimension $2 n-4$, and

$$
\left(\partial_{S p}\right)_{*}: H_{*}\left(M(1, S p(n)) ; Z_{2}\right) \rightarrow H_{*}\left(B O(1) ; Z_{2}\right)
$$

is a surjection through dimension $4 n-1$.

To prove $\partial_{u, \infty}$ is a homotopy equivalence for $k>1$, it suffices to show that it induces an isomorphism in homology with integral coefficients, because $B U(k)$ is simply connected. Moreover, we already have established that $M(k, S U) \simeq B U(k)$. Hence, to prove $\partial_{u, \infty}$ is homotopy equivalence, we need to show only that it induces a surjection in homology. To this end, we make the following observation: Taubes gluing is compatible via the Dirac maps with the Whitney sum operation. To be more precise, consider the diagram

$$
\begin{array}{ccc}
H_{*}\left(M(1, S U(n))^{k} ; Z\right) & \stackrel{\left(\partial_{u}^{k}\right)_{*}}{\longrightarrow} & H_{*}\left(B U(1)^{k} ; Z\right) \\
(\tau)_{*} \downarrow & \downarrow(w)_{*} \\
H_{*}(M(k, S U(n)) ; Z) & \stackrel{\left(\partial_{u}\right)_{*}}{\longrightarrow} & H_{*}(B U(k) ; Z),
\end{array}
$$

where $\tau$ is the Taubes gluing map and $w$ gives the Whitney sum operation on line bundles. The claim is that this diagram commutes. We will prove this shortly. For now, suppose this is the case. Then it is easy to see that $\left(\partial_{u}\right)_{*}$ is a surjection through a range: by the commutativity of the above diagrams, 
we have

$$
\left(\partial_{u}\right)_{*} \circ(\tau)_{*}=(w)_{*} \circ\left(\partial_{u}^{k}\right)_{*} \text {. }
$$

But $(w)_{*}$ is surjective, and, by the observation following the previous lemma, the map

$$
\left(\partial_{u}^{k}\right)_{*}: H_{*}\left(M(1, S U(n))^{k} ; Z\right) \rightarrow H_{*}\left(B U(1)^{k} ; Z\right)
$$

must be a surjection through dimension $2 n-4$. Thus, their composition is a surjection through dimension $2 n-4$. This forces

$$
\left(\partial_{u}\right)_{*}: H_{*}(M(k, S U(n)) ; Z) \rightarrow H_{*}\left(B U(1)^{k} ; Z\right)
$$

to be surjection through dimension $2 n-4$, and the map

$$
\left(\partial_{u, \infty}\right)_{*}: H_{*}(M(k, S U) ; Z) \rightarrow H_{*}(B U(k) ; Z)
$$

is then a surjection in all dimensions.

Therefore, to prove that $\partial_{u, \infty}$ is a homotopy equivalence, we are reduced to showing that the above diagram relating Whitney sum to Taubes gluing commutes. Essentially, this argument rests on the compatibility of Taubes gluing with the loop sum operation. Consider the diagram below.

$$
\begin{aligned}
& B U(1)^{k} \quad \stackrel{=}{\longrightarrow} \quad B U(1)^{k} \quad \stackrel{w}{\longrightarrow} \quad B U(k) \\
& \partial_{u}^{k} \uparrow \\
& \begin{array}{r}
M(1, S \\
\tau \downarrow
\end{array} \\
& M(k, S U(l)) \stackrel{i_{k, l}}{\longrightarrow} \\
& \Omega_{1}^{3} S U(l)^{k} \\
& \downarrow \\
& \Omega_{k}^{3} S U(l) \\
& \partial_{u} \downarrow \\
& B U(k) \\
& \downarrow= \\
& \downarrow= \\
& \downarrow \\
& \downarrow= \\
& \stackrel{\beta_{u(l)}, k}{\longrightarrow} \quad\{k\} \times B U \longleftarrow B U(k) \\
& =
\end{aligned}
$$
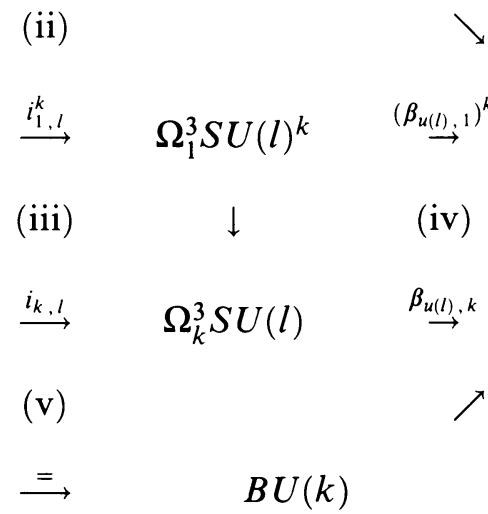

where the map $\Omega_{1}^{3} S U(l)^{k} \rightarrow \Omega_{k}^{3} S U(l)$ is given by loop sum, the map $(\{1\} \times$ $B U)^{k} \rightarrow\{k\} \times B U$ is given by the natural $\mathrm{H}$-space structure of $Z \times B U$, and all other unlabelled arrows are the natural inclusions. We show that this diagram commutes up to homotopy by showing that each of the inner diagrams commute: (i) commutes by general facts about Whitney sum; (ii) and (v) follow from the homotopy commutativity of the diagram in Proposition 8; (iii) commutes because Taubes gluing is compatible with loop sum; and (iv) commutes because $\beta_{u}$ is an $\mathrm{H}$-space map. In particular, then, we can conclude that the outer perimeter of this diagram commutes. But $B U(k) \rightarrow\{k\} \times B U$ induces an injection in homology. Thus, the two compositions forming the outer perimeter:

$$
M(1, S U(l))^{k} \stackrel{\partial_{u}^{k}}{\longrightarrow} B U(1)^{k} \stackrel{w}{\longrightarrow} B U(k)
$$

and

$$
M(1, S U(l))^{k} \stackrel{\tau}{\longrightarrow} M(k, S U(l)) \stackrel{\partial_{u}}{\longrightarrow} B U(k)
$$


induce identical maps in homology. This is exactly the claim that in homology Taubes gluing is compatible via the Dirac maps with the Whitney sum operation. Similar arguments show that the diagrams relating Whitney sum to Taubes gluing for symplectic instantons induce commutative diagrams in homology with $Z_{2}$ coefficients, and thus, that $\partial_{S p, \infty}$ induces a $Z_{2}$-homology equivalence. In particular the map $\partial_{S p}: M(k, S p(n)) \rightarrow B O(k)$ will induce a surjection in homology through dimension $4 n-1$.

To prove that the limit map $\partial_{S p, \infty}$ is in fact a homotopy equivalence, we need to examine the induced map in homology with coefficients in the rationals and the fields $Z / p$ for $p$ a prime $\neq 2$. For any spaces $X, Y$, and map $f: X \rightarrow Y$, and any coefficient ring $A$, let $(f)_{*}^{A}: H_{*}(X ; A) \rightarrow H_{*}(Y ; A)$ denote the induced map with coefficients in $A$. In the remainder of this section let $F$ stand for the field $Z / p, p \neq 2$, or the field of rational numbers. Let $\hat{M}(k, S p)$ denote the universal cover of $M(k, S p)$. Then the map $\partial_{S p}$ lifts to a map $\hat{\partial}_{S p}$ :

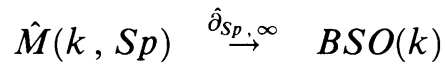

$$
\begin{aligned}
& \downarrow \\
& M(k, S p) \stackrel{\partial_{S p, \infty}}{\longrightarrow} B O(k) .
\end{aligned}
$$

Because $M(k, S p) \simeq B O(k)$ and $\hat{M}(k, S p) \simeq \widehat{B O}(k) \simeq B S O(k)$ and the map

$$
H_{*}\left(B S O(k) ; Z_{2}\right) \rightarrow H_{*}\left(B O(k) ; Z_{2}\right)
$$

is injective, we know that $\left(\hat{\partial}_{S p, \infty}\right)_{*}^{Z_{2}}$ is an isomorphism. Now suppose that $\left(\hat{\partial}_{S p, \infty}\right)_{*}^{F}$ is an isomorphism. Then by the Universal Coefficient Theorem, $\left(\hat{\partial}_{S p, \infty}\right)_{*}^{Z}$ is an isomorphism as well, and, therefore, so is $\pi_{*}\left(\hat{\partial}_{S p, \infty}\right)$, the induced map on homotopy groups. Since $\pi_{1}\left(\partial_{S p, \infty}\right)$ is an isomorphism, we must have $\pi_{i}\left(\partial_{S p, \infty}\right)$ an isomorphism for every $i$. Hence, $\partial_{S p, \infty}$ is a homotopy equivalence.

Thus, to prove that $\partial_{S p, \infty}$ is a homotopy equivalence, it suffices to prove the following lemma.

Lemma 11. $\left(\hat{\partial}_{S p, \infty}\right)_{*}^{F}$ is an isomorphism, where $F$ is any field $Z / p, p \neq 2$, or the field of rational numbers.

Proof. Identify $U(k)$ with the subset of $O(2 k)$ consisting of matrices of the form

$$
\left(\begin{array}{cc}
A & B \\
-B & A
\end{array}\right)
$$

where $A, B \in \operatorname{End}\left(R^{k}\right)$. Let $i_{u, o}: B U(k) \rightarrow B O(2 k)$ be the map induced by this natural inclusion. We first show that there exists a map $f_{1}: M(k, S U(n)) \rightarrow$ $M(2 k, S p(n))$ such that the diagram 


$$
\begin{array}{ccc}
M(k, S U(n)) & \stackrel{\partial_{u}}{\rightarrow} & B U(k) \\
f_{1} \downarrow & \downarrow i_{u, o} \\
M(2 k, S p(n)) \stackrel{\partial_{S p}}{\longrightarrow} & B O(2 k)
\end{array}
$$

induces a commutative diagram in homology with coefficients in $F$. To this end, suppose $\omega$ is a self-dual connection on the $S U(n)$ vector bundle $E_{k}$, where $c_{2}\left(E_{k}\right)=k$. Then we can define a connection $\bar{\omega}$ on the conjugate bundle $\bar{E}_{k}$. If $\omega$ is given locally by the matrix of one forms $\left(\omega_{i j}\right)$, then $\bar{\omega}$ is given locally by the matrix $\left(\bar{\omega}_{i j}\right)$. Because the transition functions for $\bar{E}_{k}$ are the conjugates of those for $E_{k},\left(\bar{\omega}_{i j}\right)$ is a well-defined connection on $\bar{E}_{k}$. Because the Hodge star operator commutes with conjugation, $\bar{\omega}$ is self-dual if $\omega$ is. We can now define a map

$$
f: M(k, S U(n)) \rightarrow M(2 k, S U(2 n))
$$

by $f(\omega)=\omega \oplus \bar{\omega}$, where $\omega \oplus \bar{\omega}$ is a self-dual connection defined on the bundle $E_{k} \oplus \bar{E}_{k}$. As we did in Section 3, we can view $S p(n)$ as a subset of $S U(2 n)$. For convenience, we choose the skew-bilinear form on $C^{2 n}$ to be given by the matrix

$$
\left(\begin{array}{cc}
0 & -I_{n} \\
I_{n} & 0
\end{array}\right) \text {. }
$$

Then $S p(n) \subset S U(2 n)$ may be viewed as matrices of the form

$$
\left(\begin{array}{cc}
A & -\bar{B} \\
B & \bar{A}
\end{array}\right)
$$

where $A, B \in \operatorname{End}\left(C^{n}\right)$. Consequently, the map $S U(n) \rightarrow S U(2 n)$ given by

$$
A \mapsto\left(\begin{array}{cc}
A & 0 \\
0 & \bar{A}
\end{array}\right)
$$

factors through $S p(n)$. Thus, by considering its transition functions we see that $E_{k} \oplus \bar{E}_{k}$ is an $S p(n)$ bundle. Furthermore, because $\omega \oplus \bar{\omega}$ is a self-dual connection given locally by a matrix of one forms with values in the Lie algebra of $S p(n) \subset S U(2 n)$, the map $f$ factors as

$$
M(k, S U(n)) \rightarrow M(2 k, S p(n)) \rightarrow M(2 k, S U(2 n)) .
$$

We will denote the first map in this composition by $f_{1}$. Now consider the diagram

$$
\begin{array}{ccc}
M(k, S U(n)) & \stackrel{\partial_{u}}{\longrightarrow} & B U(k) \\
f_{1} \downarrow & \downarrow i_{u, o} \\
M(2 k, S p(n)) & \stackrel{\partial_{S p}}{\longrightarrow} & B O(2 k) \\
\downarrow & & \downarrow i_{o, u} \\
M(2 k, S U(2 n)) & \stackrel{\partial_{u}}{\longrightarrow} & B U(2 k)
\end{array}
$$


where $i_{o, u}$ is induced by the natural inclusion $O(2 k) \hookrightarrow U(2 k)$. The bottom square homotopy commutes because given a complex bundle $V$ with a real structure given by a real sub-bundle $V_{+}$, there is an isomorphism $V \cong V_{+} \otimes$ $C$. The large outer square also homotopy commutes: if we let $K$ denote the "kernel" bundle of the Dirac operators coupled to instantons, or in other words, the bundle classified by the Dirac map $\partial_{u}: M(k, S U(n)) \rightarrow B U(k)$, then both compositions, $i_{o, u} \circ i_{u, o} \circ \partial_{u}$ and $\partial_{u} \circ f$, classify the isomorphism class of the $2 k$ dimensional bundle $K \oplus \bar{K}$ over $M(k, S U(n))$. Finally, we point out that the map $\left(i_{o}, u\right)_{*}^{F}$ is an injection. This implies that the upper square of the diagram induces a diagram in homology with coefficients in $F$ which commutes. Hence we have the commutative diagram

$$
\begin{array}{ccc}
H_{*}(M(k, S U(n)) ; F) & \stackrel{\left(\partial_{u}\right)_{*}^{F}}{\longrightarrow} & H_{*}(B U(k) ; F) \\
\left(f_{1}\right)_{*}^{F} \downarrow & \downarrow\left(i_{u, o}\right)_{*}^{F} \\
H_{*}(M(2 k, S p(n)) ; F) \stackrel{\left(\partial_{S p}\right)_{*}^{F}}{\longrightarrow} & H_{*}(B O(2 k) ; F) .
\end{array}
$$

Because $\left(\partial_{u}\right)_{*}^{F}$ and $\left(i_{u, o}\right)_{*}^{F}$ are both surjections through dimension $2 n-4$, $\left(\partial_{S p}\right)_{*}^{F}$ must be a surjection through that range as well. Hence, the limit map

$$
\left(\partial_{S p, \infty}\right)_{*}^{F}: H_{*}(M(2 k, S p) ; F) \rightarrow H_{*}(B O(2 k) ; F)
$$

must be a surjection. Since $M(2 k, S p) \simeq B O(2 k),\left(\partial_{S p, \infty}\right)_{*}^{F}$ must be an isomorphism. Now, $\pi_{1}(B U(k)) \cong \pi_{1}(M(k, S U(n)))=0$ (for $n>2$ ), so we can lift $f_{1}$ and $i_{u, o}$ to the universal covers to obtain the homotopy commutative diagram

$$
\begin{aligned}
& M(k, S U(n))=M(k, S U(n)) \stackrel{\partial_{u}}{\longrightarrow} B U(k)=B U(k) \\
& \hat{f}_{1} \downarrow \quad f_{1} \downarrow \quad \downarrow i_{u, o} \quad \downarrow \hat{i}_{u, o} \\
& \hat{M}(2 k, S p(n)) \quad \rightarrow \quad M(2 k, S p(n)) \stackrel{\partial_{S p}}{\longrightarrow} B O(2 k) \leftarrow B S O(2 k)
\end{aligned}
$$

Again, because $\pi_{1}(\hat{M}(2 k, S p(n)))=0$, we can lift the composition

$$
\hat{M}(2 k, S p(n)) \rightarrow M(2 k, S p(n)) \rightarrow B O(2 k),
$$

to obtain $\hat{\partial}_{S p}: \hat{M}(2 k, S p(n)) \rightarrow B S O(2 k)$. Together with the homotopy commutativity of the above diagram we get the homotopy commutative diagram

$$
\begin{array}{ccc}
M(k, S U(n)) & \stackrel{\partial_{u}}{\longrightarrow} & B U(k) \\
\hat{f}_{1} \downarrow & \downarrow \hat{i}_{u, o} \\
\hat{M}(2 k, S p(n)) & \stackrel{\hat{\partial}_{S p}}{\longrightarrow} & B S O(2 k) .
\end{array}
$$

Since $\hat{i}_{u, o}$ and $\partial_{u}$ both induce $F$-homology surjections through dimension $2 n-4$, so does $\hat{\partial}_{S p}$. Because $\hat{M}(2 k, S p) \simeq B S O(2 k)$, the limit map $\left(\hat{\partial}_{S p, \infty}\right)_{*}^{F}$ 
must be an isomorphism. This proves the lemma when the second Chern class is even. For odd Chern class, we consider the diagram

$$
\begin{array}{ccc}
M(2 k, S p(n)) & \stackrel{\partial_{S p}}{\longrightarrow} & B O(2 k) \\
\tau \downarrow & \downarrow w \\
M(2 k+1, S p(n)) & \stackrel{\partial_{S p}}{\longrightarrow} & B O(2 k+1),
\end{array}
$$

where $\tau$ is defined by gluing with a charge-one instanton and $w$ is induced by the natural inclusion $O(2 k) \hookrightarrow O(2 k+1)$. It was shown in Section 5 that when viewed on the level of homology, Taubes gluing is compatible via the Dirac map with the Whitney sum operation on bundles. The same argument proves that the above diagram induces a commutative diagram in homology with coefficients in $F$. We have already shown that $\left(\partial_{S p}\right)_{*}^{F}$ is a surjection through a range, and it is well known that $(w)_{*}^{F}$ is an isomorphism. Thus, by the same arguments as before we can conclude that the limit map $\left(\partial_{S p, \infty}\right)_{*}^{F}: H_{*}(M(2 k+1, S p) ; F) \rightarrow H_{*}(B O(2 k+1) ; F)$ must be an isomorphism. Finally, by considering the diagram

$$
\begin{array}{ccc}
\hat{M}(2 k+1, S p) & \stackrel{\hat{\partial}_{S p, \infty}}{\longrightarrow} & B S O(2 k+1) \\
\downarrow & \downarrow \\
M(2 k+1, S p) \stackrel{\partial_{S p, \infty}}{\longrightarrow} & B O(2 k+1),
\end{array}
$$

recalling that $H_{*}(B S O(2 k+1) ; F) \cong H_{*}(B O(2 k+1) ; F)$, and that $\hat{M}(2 k+1, S p) \simeq B S O(2 k+1)$, one sees that $\hat{\partial}_{S p, \infty}$ must induce an $F$ homology equivalence. This finishes the lemma, and also the proof that $\partial_{S p, \infty}$ is a homotopy equivalence.

\section{THE hoMOlogy OF THE DiRAC MAPS}

Up to this point we have focused our attention on the Dirac maps on the limit spaces $M(k, S U)$ and $M(k, S p)$. In this section, we examine the Dirac maps on the finite dimensional $M(k, S U(l))$ and $M(k, S p(l))$ and compute the images in homology of the maps $\partial_{u}: M(k, S U(l)) \rightarrow B U(k)$ and $\partial_{S p}$ : $M(k, S p(l)) \rightarrow B O(k)$. We first deal with unitary connections.

Recall that there is a homotopy commutative diagram

$$
\begin{array}{ccc}
M(k, S U(l)) & \stackrel{\partial_{u}}{\longrightarrow} & B U(k) \\
i_{k, l} \downarrow & & \downarrow \\
\Omega_{k}^{3} S U(l) \stackrel{\beta_{u(l), k}}{\longrightarrow} & \{k\} \times B U .
\end{array}
$$

Define $j_{k, l}: M(k, S U(l)) \rightarrow\{k\} \times B U$ to be either composition in the above diagram, and let $J(k, l) \subset H_{*}(\{k\} \times B U ; Z)$ be the intersection of the images 
of the maps

$$
H_{*}(B U(k) ; Z)
$$

$$
H_{*}\left(\Omega_{k}^{3} S U(l) ; Z\right) \stackrel{\left(\beta_{u(l), k}\right) *}{\longrightarrow} H_{*}(\{k\} \times B U ; Z) .
$$

Notice that by the commutativity of the square diagram above, the image of $\left(j_{k, l}\right)_{*}$ is contained in $J(k, l)$. Let $b_{i} \in H_{2 i}(\{1\} \times B U ; Z)$ be the image of a generator of $H_{2 i}(B U(1) ; Z)$ under the map induced by the natural inclusion $B U(1) \hookrightarrow\{1\} \times B U$. Then we can write $H_{*}(Z \times B U ; Z)=$ $Z\left[b_{0}, b_{0}^{-1}, b_{1}, b_{2}, \ldots\right]$. For a monomial $b_{j_{1}}^{i_{1}} \cdots b_{j_{r}}^{i_{r}}$ define length $\left(b_{j_{1}}^{i_{1}} \cdots b_{j_{r}}^{i_{r}}\right)=$ $\sum_{s} i_{s}$. We now can state one of our main theorems.

Theorem 12. The image of $\left(j_{k, l}\right)_{*}$ coincides with the set $J(k, l)$. In fact this set is equal to is the set of all polynomials in $b_{0}, b_{1}, \ldots, b_{l-2}$ which are a sum of monomials of length $k$.

The strategy for proving Theorem 12 is as follows: first we work rationally to calculate the image in rational homology of the map $\left(\beta_{u(l)}\right)_{*}^{Q}$. Although this could be accomplished without reference to instantons, our proof shows that the computation follows immediately from the compatibility of Taubes gluing with the Whitney sum operation on line bundles. Because $Z \times B U$ has no torsion in its homology, the image of $\left(\beta_{u(l)}\right)_{*}^{Q}$ gives essentially an "upper bound" for the image of $\left(\beta_{u(l)}\right)_{*}^{Z}$ consisting of polynomials in $b_{0}, b_{0}^{-1}, b_{1}, \ldots, b_{l-2}$. Finally, by again exploiting the compatibility of Taubes gluing with Whitney sum, we show that any such polynomial which is also in the image of $H_{*}(B U(k) ; Z)$, must in fact have a pre-image in $H_{*}\left(M(1, l)^{k} ; Z\right)$ and, hence, must be in the image of the map $\left(j_{k, l}\right)_{*}$.

Our first lemma is fairly standard.

Lemma 13. $\Omega_{0}^{3} S U(l)$ is rationally homotopy equivalent to $\Omega S U(l-1)$. Thus, there exist $d_{0}, d_{0}^{-1}, d_{1}, \ldots, d_{l-2}, d_{i} \in H_{2 i}\left(\Omega^{3} S U(l) ; Q\right)$, such that

$$
H_{*}\left(\Omega^{3} S U(l) ; Q\right)=Q\left[d_{0}, d_{0}^{-1}, \ldots, d_{l-2}\right] .
$$

Proof. There are rational homotopy equivalences as $\mathrm{H}$-spaces

$$
S U(l) \simeq_{Q} S^{3} \times S^{5} \times \cdots \times S^{2 l-1} \simeq_{Q} K(Q, 3) \times \cdots \times K(Q, 2 l-1) .
$$

Thus,

$\Omega_{0}^{3} S U(l) \simeq_{Q} K(Q, 2) \times \cdots \times K(Q, 2 l-4) \simeq_{Q} \Omega(K(Q, 3) \times \cdots \times K(Q, 2 l-3))$

and we see that $\Omega_{0}^{3} S U(l-1) \simeq_{Q} \Omega S U(l-1)$. Now, $H_{*}(\Omega S U(l-1) ; Q)$ is known to be a polynomial algebra on generators $e_{i} \in H_{2 i}$ for $1 \leq i \leq l-2$. Therefore, there are elements $d_{0}, d_{0}^{-1}, d_{1}, \ldots, d_{l-2}, d_{i} \in H_{2 i}\left(\Omega^{3} S U(l) ; Q\right)$, such that $H_{*}\left(\Omega^{3} S U(l) ; Q\right)=Q\left[d_{0}, d_{0}^{-1}, d_{1}, \ldots, d_{l-2}\right]$. This finishes the first lemma.

Define $\tilde{b}_{i} \in H_{2 i}(Z \times B U ; Q)$ to be the image of $b_{i}$ under the natural map

$$
H_{*}(Z \times B U ; Z) \rightarrow H_{*}(Z \times B U ; Q) .
$$


Lemma 14. The image of the map

$$
\left(\beta_{u(l)}\right)_{*}^{Q}: H_{*}\left(\Omega^{3} S U(l) ; Q\right) \rightarrow H_{*}(Z \times B U ; Q)
$$

is the polynomial subalgebra $Q\left[\tilde{b}_{0}, \tilde{b}_{0}^{-1}, \tilde{b}_{1}, \ldots, \tilde{b}_{l-2}\right]$.

Proof. Consider the following diagram which we know commutes in homology:

$$
\begin{array}{ccc}
\amalg_{k \geq 0}(M(1, S U(l)))^{k} & \stackrel{\bigsqcup_{k} \partial_{u}^{k}}{\longrightarrow} & \amalg_{k \geq 0}(B U(1))^{k} \\
\tau \downarrow & & \downarrow \\
\amalg_{k \geq 0} M(k, S U(l)) & \stackrel{\bigsqcup_{k} \partial_{u}}{\longrightarrow} & \amalg_{k \geq 0} B U(k) \\
\downarrow & & \downarrow \\
\Omega^{3} S U(l) & \stackrel{\beta_{u(l)}}{\longrightarrow} & Z \times B U .
\end{array}
$$

Let $z_{i} \in H_{2 i}(M(1, S U(l)) ; Q)$ be a generator for $0 \leq i \leq l-2$, and suppose that $a_{i} \in H_{2 i}\left(\Omega^{3} S U(l) ; Q\right)$ is the image of $z_{i}$ under the homology map induced by

$$
M(1, S U(l)) \rightarrow \Omega_{1}^{3} S U(l) \rightarrow \Omega^{3} S U(l) .
$$

We claim that $Q\left[a_{0}^{-1}, a_{0}, a_{1}, \ldots, a_{l-2}\right]=H_{*}\left(\Omega^{3} S U(l) ; Q\right)$. By dimension considerations and the preceding lemma, it suffices to show that no polynomial in the $a_{i}$ is zero. So suppose that $P\left(a_{0}, a_{1}, \ldots, a_{l-2}\right)=0$. (We can assume, by multipiying by a large enough power of $a_{0}$ that $a_{0}^{-1}$ does not occur.) Consider the image of $z_{i}$ under the homology map induced by

$$
M(1, S U(l)) \rightarrow B U(1) \rightarrow\{1\} \times B U .
$$

By Lemma 6, $z_{i}$ maps to $\tilde{b}_{i} \in H_{2 i}(Z \times B U ; Q)$. Thus, $\left(\beta_{u(l)}\right)_{*}$ maps $a_{i}$ to $\tilde{b}_{i}$, and so $P\left(a_{0}, \ldots, a_{l-2}\right)$ maps to $P\left(\tilde{b}_{0}, \tilde{b}_{1}, \ldots, \tilde{b}_{l-2}\right)$, which is obviously non-zero in $H_{*}(Z \times B U ; Q)$. Consequently, $P\left(a_{0}, \ldots, a_{l-2}\right) \neq 0$ and the claim is proved. But we have also shown that $a_{i} \mapsto \tilde{b}_{i}$; hence, the image of $\left(\beta_{u(l)}\right)_{*}^{Q}$ is the polynomial algebra $Q\left[\tilde{b}_{0}, \tilde{b}_{0}^{-1}, \tilde{b}_{1}, \ldots, \tilde{b}_{l-2}\right]$, which finishes the lemma.

Because $H_{*}(B U ; Z)$ is torsion free, the image of the integral homology map $\left(\beta_{u(l)}\right)_{*}: H_{*}\left(\Omega^{3} S U(l) ; Z\right) \rightarrow H_{*}(Z \times B U ; Z)$ must be contained in the polynomial algebra $Z\left[b_{0}, b_{0}^{-1}, b_{1}, \ldots, b_{l-2}\right]$. The image of $\left(\beta_{u(l), k}\right)_{*}$ restricted to the $k$ th component, $\Omega_{k}^{3} S U(l)$, is then a subset of the polynomials in $b_{0}, b_{0}^{-1}, \ldots, b_{l-2}$ such that each term has length $k$. Furthermore, by considering the surjective map $H_{*}\left(B U(1)^{k} ; Z\right) \rightarrow H_{*}(B U(k) ; Z)$, one sees that the image of the inclusion $H_{*}(B U(k) ; Z) \hookrightarrow H_{*}(Z \times B U ; Z)$ consists of all polynomials in $b_{0}, b_{1}, b_{2}, \ldots$ with terms having length exactly $k$. Hence, $J(k, l)$ is contained in the set of all polynomials in $b_{0}, b_{1}, \ldots, b_{l-2}$ with terms of length $k$. (In fact our proof of Theorem 12 will show that $J(k, l)$ is exactly equal to this set of polynomials.) We now show that any such polynomial is in the image of $H_{*}(M(k, S U(l)) ; Z)$. We prove this by showing that, in fact, 
any element of $J(k, l)$ is contained in the image of the homology of the glued charge-one instantons. We have the following commutative diagram:

$$
\begin{array}{ccc}
H_{*}\left(\left(C P^{l-1}\right)^{k} ; Z\right) & \longrightarrow & H_{*}\left(\left(C P^{\infty}\right)^{k} ; Z\right) \\
(\pi)_{*} \uparrow & \downarrow= \\
H_{*}\left(M(1, S U(l))^{k} ; Z\right) & \stackrel{\left(\partial_{u}^{k}\right)_{*}}{\longrightarrow} & H_{*}\left(B U(1)^{k} ; Z\right) \\
(\tau)_{*} \downarrow & \downarrow \\
H_{*}(M(k, S U(l)) ; Z) & \stackrel{\left(\partial_{u}\right)_{*}}{\longrightarrow} & H_{*}(B U(k) ; Z) \\
\downarrow & & \downarrow \\
H_{*}\left(\Omega_{k}^{3} S U(l) ; Z\right) & \stackrel{\left(\beta_{u(l), k}\right)_{*}}{\longrightarrow} & H_{*}(\{k\} \times B U ; Z)
\end{array}
$$

Since the map $H_{*}(M(1, S U(l)) ; Z) \rightarrow H_{*}\left(C P^{l-1} ; Z\right)$ is a surjection through dimension $2 l-4$, and for any $0 \leq i \leq l-2, b_{i}$ is in the image of a generator of $H_{2 i}\left(C P^{l-1} ; Z\right)$, we see that any polynomial in $b_{0}, b_{1}, \ldots, b_{l-2}$ which has terms of length $k$ is in the image of $H_{*}\left(M(1, S U(l))^{k} ; Z\right)$. Thus, by the commutativity of the above diagram, any such polynomial is in the image of $H_{*}(M(k, S U(l)))$. This completes the proof of the theorem.

Note that this argument also shows that any element which is a polynomial in $b_{0}, \ldots, b_{l-2}$ having terms of length $k$ is in $J(k, l)$. In fact since $M(1, S U(l))$ is homotopy equivalent to a $S^{2 l-3}$-bundle over $C P^{l-1}$, the natural inclusion $C P^{l-2} \hookrightarrow C P^{l-1}$ lifts, uniquely up to homotopy, to $M(1, S U(l))$. Hence there are maps

$$
\left(C P^{l-2}\right)^{k} \rightarrow M(1, S U(l))^{k} \rightarrow M(k, l) \stackrel{j_{k, l}}{\rightarrow}\{k\} \times B U
$$

as well as a natural map

$$
\delta:\left(C P^{l-2}\right)^{k} \rightarrow\{k\} \times B U
$$

defined as the composition

$$
\left(C P^{l-2}\right)^{k} \hookrightarrow\left(C P^{l-1}\right)^{k} \hookrightarrow B U(1)^{k} \rightarrow B U(k) \hookrightarrow\{k\} \times B U
$$

The proof of the previous theorem shows that these maps induce identical homology maps, and that $\operatorname{Image}\left((\delta)_{*}\right)=\operatorname{Image}\left(\left(j_{k, l}\right)_{*}\right)=J(k, l)$. 


\section{THE HOMOLOGY OF THE SYMPLECTIC DiRAC MAP}

We now turn olir attention to the symplectic case. Recall the diagram

$$
\begin{array}{ccc}
M(k, S p(l)) & \stackrel{\partial_{S p}}{\longrightarrow} & B O(k) \\
i_{k, l} \downarrow & & \downarrow \\
\Omega_{k}^{3} S p(l) \stackrel{\beta_{S p(l), k}}{\longrightarrow}\{k\} \times B O .
\end{array}
$$

Let $j_{k, l}: M(k, S p(l)) \rightarrow\{k\} \times B O$ be either composition in the above diagram, and let $J_{S p}(k, l)$ be the intersection of the images of the maps

$$
\begin{gathered}
H_{*}\left(B O(k) ; Z_{2}\right) \\
\downarrow \\
H_{*}\left(\Omega_{k}^{3} S p(l) ; Z_{2}\right) \longrightarrow H_{*}\left(\{k\} \times B O ; Z_{2}\right) .
\end{gathered}
$$

Theorem 15. The image of the map $\left(j_{k, l}\right)_{*}^{Z_{2}}$ is precisely the set $J_{S p}(k, l)$.

As in the unitary case, the proof of Theorem 15 consists of two parts. First we show that there is a natural map $\Omega^{3} \Sigma^{3}\left(R P_{+}^{4 l-1}\right) \rightarrow \Omega^{3} S p(l)$ which induces a surjection in homology with $Z_{2}$ coefficients, and such that the composition

$$
H_{*}\left(\Omega^{3} \Sigma^{3}\left(R P_{+}^{4 l-1}\right) ; Z_{2}\right) \rightarrow H_{*}\left(\Omega^{3} S p(l) ; Z_{2}\right) \rightarrow H_{*}\left(Z \times B O ; Z_{2}\right)
$$

is computable in terms of natural ring generators for $H_{*}\left(Z \times B O ; Z_{2}\right)$. We can then compute $J_{S p}(k, l)$. This requires using results of $\mathrm{F}$. Cohen [CLM] on the homology of iterated loop spaces, as well as calculations of Kochman [Ko] on how certain Dyer-Lashof operations act on the natural ring generators of $H_{*}\left(Z \times B O ; Z_{2}\right)$. Second, we show that any such element in $J_{S p}(k, l)$ has a pre-image in the homology of the glued charge-one instantons.

Unlike the unitary case, however, we now require a more delicate gluing of instantons using configurations of points with labels in $M(k, S p(l))$. More precisely, let

$$
C_{3}(k)=\left\{\left(x_{1}, \ldots, x_{k}\right) \in\left(R^{3}\right)^{k} \mid x_{i} \neq x_{j}, \text { if } i \neq j\right\}
$$

denote $k$-tuples of distinct points in $R^{3}$. Using results of Taubes [T1], Boyer and Mann [BM] show that there exists maps for all $k \geq 1$

$$
\tau_{k}: C_{3}(k) \times_{\Sigma_{k}}(M(1, S p(l)))^{k} \rightarrow M(k, S p(l)),
$$

which make the following diagram homotopy commute:

$$
\begin{array}{ccc}
C_{3}(k) \times \Sigma_{k}(M(1, S p(l)))^{k} & \stackrel{\tau_{k}}{\rightarrow} & M(k, S p(l)) \\
1 \times \Sigma_{k}\left(i_{1, l}\right)^{k} \downarrow & & \downarrow i_{k, l} \\
C_{3}(k) \times \Sigma_{k}\left(\Omega_{1}^{3} S p(l)\right)^{k} & \rightarrow & \Omega_{k}^{3} S p(l),
\end{array}
$$

where the bottom horizontal map is the standard $C_{3}$-operad map corresponding to the standard three-fold loop sum structure on $\Omega^{3} S p(l)$. Recall that for 
unitary connections, the compatibility of the gluing maps with the loop sum map, as well as the compatibility of the Dirac map with Bott periodicity, implied the commutativity of

$$
\begin{array}{ccc}
H_{*}\left(M(1, S U(l))^{k} ; Z\right) & \stackrel{\left(\partial_{u}^{k}\right)_{*}}{\longrightarrow} & H_{*}\left(B U(1)^{k} ; Z\right) \\
(\tau)_{*} \downarrow & & \downarrow(w)_{*} \\
H_{*}(M(k, S U(l)) ; Z) & \stackrel{\left(\partial_{u}\right)_{*}}{\longrightarrow} & H_{*}(B U(k) ; Z) \\
\downarrow & & \downarrow \\
H_{*}\left(\Omega_{k}^{3} S U(l) ; Z\right) & \stackrel{\left(\beta_{u(l), k}\right)_{*}}{\longrightarrow} & H_{*}(\{k\} \times B U ; Z) .
\end{array}
$$

We now show that an analogous diagram, where we glue with configurations of points with labels in $M(1, S p(l))$, also commutes by considering the following diagram (cf. Section 5, proof of theorem 9):

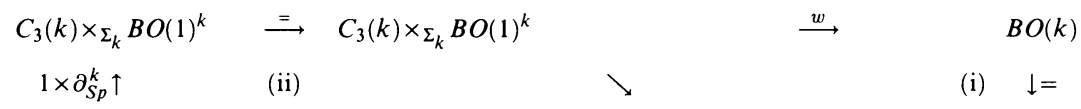

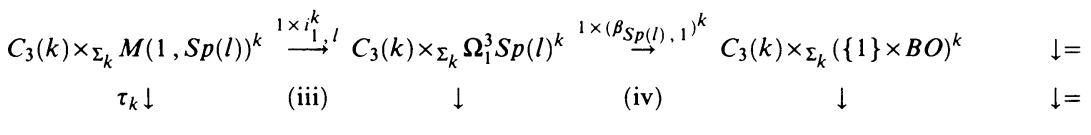

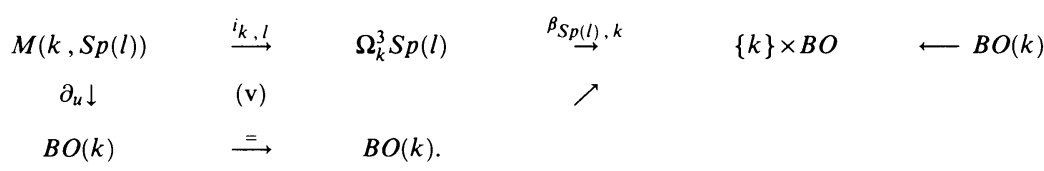

As in Section 5, squares (i), (ii), and (v) commute up to homotopy. By the compatibility of the maps $\tau_{k}$ with the $C_{3}$-operad structure on $\Omega^{3} S p(l)$, square (iii) also commutes up to homotopy. Finally, square (iv) commutes up to homotopy because $\beta_{S p}$ is an infinite loop equivalence, and, therefore, also respects the operad structure. Because $B O(k) \rightarrow B O$ induces an injection in homology with $Z_{2}$ coefficients, we see there is a commutative diagram

$$
\begin{array}{ccc}
H_{*}\left(C_{3}(j) \times{ }_{\Sigma_{j}} M(1, S p(l))^{j} ; Z_{2}\right) & \stackrel{1 \times\left(\partial_{S_{p}^{j}}^{j}\right)_{*}^{Z_{2}}}{\longrightarrow} & H_{*}\left(C_{3}(j) \times \Sigma_{j} B O(1)^{j} ; Z_{2}\right) \\
\left(\tau_{j}\right)_{*}^{Z_{2}} \downarrow & & \downarrow \\
H_{*}\left(M(j, S p(l)) ; Z_{2}\right) & \stackrel{\left(\partial_{S p}\right)_{*}^{Z_{2}}}{\longrightarrow} & H_{*}\left(B O(j) ; Z_{2}\right) .
\end{array}
$$


Summing over the Chern class and identifying $B O(1)$ with $R P^{\infty}$, we have shown that the diagram

$$
\begin{array}{ccc}
\amalg_{1 \leq j \leq k} C_{3}(j) \times_{\Sigma_{j}}(M(1, S p(l)))^{j} & \stackrel{\amalg_{j} 1 \times \partial_{S p}^{j}}{\longrightarrow} & \amalg_{1 \leq j \leq k} C_{3}(j) \times_{\Sigma_{j}}\left(R P^{\infty}\right)^{j} \\
\amalg_{j} \tau_{j} \downarrow & \bigsqcup_{j} \partial_{S p} & \downarrow \\
\coprod_{1 \leq j \leq k} M(j, S p(l)) & & \amalg_{1 \leq j \leq k} B O(j) \\
\downarrow & & \downarrow \\
\Omega^{3} S p(l) & \stackrel{\beta_{S p(l)}}{\longrightarrow} & Z \times B O .
\end{array}
$$

induces a commutative diagram in homology with $Z_{2}$ coefficients.

A small change of notation will be useful. For a basepointed space $X$ let

$$
F_{k}(X)=\coprod_{0 \leq j \leq k} C_{3}(j) \times \Sigma_{j} X^{j} / \sim
$$

where

$$
\left(r_{1}, \ldots, r_{i}, \ldots, r_{j} ; x_{1}, \ldots, x_{i}, \ldots, x_{j}\right)
$$

is identified with

$$
\left(r_{1}, \ldots, r_{i-1}, r_{i+1}, \ldots, r_{j} ; x_{1}, \ldots, x_{i-1}, x_{i+1}, \ldots, x_{j}\right)
$$

if $x_{i}=*$, and with the convention that $C_{3}(0) \times \Sigma_{0} X^{0}$ represents the basepoint. There are natural maps $F_{k}(X) \rightarrow \Omega^{3} \Sigma^{3}(X)$, and May [M] has shown that if $X$ is path connected then $\Omega^{3} \Sigma^{3} X$ is weakly homotopy equivalent to $\lim _{k \rightarrow \infty} F_{k}(X)$. For an unbased space $X$, define $X_{+}$to be $X$ union a disjoint basepoint. From the definitions we have that for any unbased space $X$,

$$
F_{k}\left(X_{+}\right) \cong \coprod_{0 \leq j \leq k} C_{3}(j) \times \Sigma_{j} X^{j}
$$

Therefore, we may rewrite the diagram above as

$$
\begin{array}{ccc}
F_{k}\left(M(1, S p(l))_{+}\right) & \longrightarrow & F_{k}\left(R P_{+}^{\infty}\right) \\
\downarrow & \downarrow \\
\amalg_{0 \leq j \leq k} M(j, S p(l)) & \longrightarrow & \amalg_{0 \leq j \leq k} B O(j) \\
\downarrow & & \downarrow \\
\Omega^{3} S p(l) & \longrightarrow & Z \times B O .
\end{array}
$$

The strategy in proving Theorem 15 is now clear. We shall show that any element in $J_{S p}(k, l) \subset H_{*}\left(Z \times B O ; Z_{2}\right)$ has a pre-image in

$$
H_{*}\left(F_{k}\left(M(1, S p(l))_{+}\right) ; Z_{2}\right) .
$$


First, however, we need to understand the image of the map

$$
H_{*}\left(\Omega^{3} S p(l) ; Z_{2}\right) \rightarrow H_{*}\left(Z \times B O ; Z_{2}\right) .
$$

Recall that $i_{1, l}: M(1, S p(l)) \rightarrow \Omega_{1}^{3} S p(l)$ is the natural inclusion of based gauge equivalence classes of charge-one instantons into the space of based gauge equivalence classes of all charge-one connections. To obtain a basepoint preserving map, we can extend $i_{1, l}$ to the map $i_{1, l}: M(1, S p(l))_{+} \rightarrow \Omega^{3} S p(l)$, where + is mapped to the constant map in $\Omega_{0}^{3} S p(l)$. This allows us to adjoint the map, to get

$$
\operatorname{ad}\left(i_{1, l}\right): \Sigma^{3}\left(M(1, S p(l))_{+}\right) \rightarrow S p(l) .
$$

Our goal is to show that the iterated loop map

$$
\Omega^{3} \operatorname{ad}\left(i_{1, l}\right): \Omega^{3} \Sigma^{3}\left(M(1, S p(l))_{+}\right) \rightarrow \Omega^{3} S p(l)
$$

induces a surjection in $Z_{2}$-homology. We will accomplish this by first showing that the composition

$$
\Sigma^{3}\left(M(1, S p(l))_{+}\right) \stackrel{\operatorname{ad}\left(i_{1, l}\right)}{\longrightarrow} S p(l) \stackrel{p}{\longrightarrow} S^{4 l-1},
$$

where $p$ is projection onto the last column, induces a surjection in homology with $Z_{2}$ coefficients. To this end we review a useful geometric description given by Boyer, Mann and Waggoner [BMW] of the map ad $\left(i_{1, l}\right)$. First, view $S p(1) \subset S p(l)$ as quaternionic matrices of the form

$$
\left(\begin{array}{cc}
\alpha & 0 \\
0 & I_{l-1}
\end{array}\right)
$$

where $\alpha \in S p(1)$. The centralizer of $S p(1)$ in $S p(l)$ is the set

$$
\left\{\left(\begin{array}{cc}
a & 0 \\
0 & a A
\end{array}\right): a \in Z_{2}, A \in S p(l-1)\right\}=Z_{2} \times S p(l-1) .
$$

Define the map $k: S p(1) \times S p(l) \rightarrow S p(l)$ by $k(\alpha, \beta)=\beta \alpha \beta^{-1}$. By the previous remarks, $k$ descends to a map

$$
k: S p(1) \times S p(l) /\left(Z_{2} \times S p(l-1)\right) \rightarrow S p(l) .
$$

In fact, since $k(1, \beta)=1$ for any $\beta \in S p(l), k$ is well-defined as a map

$$
\begin{aligned}
k: & \left(S p(1) \times S p(l) /\left(Z_{2} \times S p(l-1)\right)\right) /\left(\{1\} \times\left(S p(l) /\left(Z_{2} \times S p(l-1)\right)\right)\right) \\
& \rightarrow S p(l) .
\end{aligned}
$$

The projection $p$ gives a homeomorphism

$$
S p(l) /\left(Z_{2} \times S p(l-1)\right) \cong R P^{4 l-1},
$$

and for any space $X$,

$$
S p(1) \times X /\{1\} \times X \cong \Sigma^{3}\left(X_{+}\right) .
$$


Thus, $k$ defines a map $k: \Sigma^{3}\left(R P_{+}^{4 l-1}\right) \rightarrow S p(l)$. Now recall Lemma 6 which showed that $M(1, S p(l)) \simeq R P^{4 l-1}$. Boyer, Mann and Waggoner [BMW] exhibit a particular homotopy equivalence $h: R P^{4 l-1} \rightarrow M(1, S p(l))$ which makes the following diagram commute:

$$
\begin{array}{ccc}
\Sigma^{3}\left(R P_{+}^{4 l-1}\right) & \stackrel{\Sigma^{3}\left(h_{+}\right)}{\longrightarrow} & \Sigma^{3}\left(M(1, S p(l))_{+}\right) \\
k \searrow & \downarrow \operatorname{ad}\left(i_{1, l}\right) \\
& & S p(l) .
\end{array}
$$

From this point forward, we will identify $R P^{4 l-1}$ with $M(1, S p(l))$ via $h$, and thus view $\operatorname{ad}\left(i_{1, l}\right)$ as a map $\Sigma^{3}\left(R P_{+}^{4 l-1}\right) \rightarrow S p(l)$. Our choice of homotopy equivalence $M(1, S p(l)) \simeq R P^{4 l-1}$ can be somewhat arbitrary because any two must induce identical maps in homology with $Z_{2}$ coefficients, and this will suffice for our purposes.

It will be useful later to note that Boyer, Mann, and Waggoner's equivalence of $M(1, S p(l))$ with the quotient of $S p(l)$ by the centralizer of $S p(1)$ is quite general. For example, they also show that there is a homotopy equivalence

$$
M(1, S U(l)) \simeq S U(l) / U(l-2),
$$

where $U(l-2)$ is identified with the centralizer of $S U(2)$ inside $S U(l)$, namely the set of matrices of the form

$$
\left(\begin{array}{lll}
a & 0 & 0 \\
0 & a & 0 \\
0 & 0 & A
\end{array}\right)
$$

where $A \in U(l-2)$ and $\operatorname{det}(A)=a^{-2}$. Furthermore, they show that via the above homotopy equivalence, the adjoint of the natural inclusion $M(1, S U(l))$ $\hookrightarrow \Omega_{1}^{3} S U(l)$, may be viewed as a map

$$
k_{u}: S U(2) \times S U(l) \rightarrow S U(l)
$$

defined by conjugation exactly as in the $S p(l)$ case.

Lemma 16. The composition $\Sigma^{3}\left(R P_{+}^{4 l-1}\right) \rightarrow S p(l) \rightarrow S^{4 l-1}$ induces a surjection in homology with $Z_{2}$ coefficients.

Proof. Although there will be some details to check, the essence of the argument is simply to view $S p(l)$ instantons as $S U(2 l)$ instantons, verify that there is a commutative diagram

$$
\begin{array}{ccccc}
\Sigma^{3}\left(M(1, S p(l))_{+}\right) & \longrightarrow & S p(l) & \stackrel{p}{\longrightarrow} & S^{4 l-1} \\
\downarrow & & \downarrow & & \downarrow= \\
\Sigma^{3}\left(M(1, S U(2 l))_{+}\right) & \rightarrow & S U(2 l) & \stackrel{p}{\longrightarrow} & S^{4 l-1}
\end{array}
$$

show that the left vertical map induces a surjection in dimension $4 l-1$ in $Z_{2}$-homology, then employ the fact proved by Boyer, Mann, and Waggoner that the bottom composition induces a surjection in homology with $Z_{2}$ coefficients. 
First we make some definitions. Recall that

$$
S U(2 l) / U(2 l-2) \simeq M(1, S U(2 l))
$$

and $M(1, S U(2 l))$ fibers over $C P^{2 l-1}$ with fiber $S^{4 l-3}$. The inclusion of the $4 l-4$ skeleton $C P^{2 l-2} \hookrightarrow C P^{2 l-1}$ lifts to a map

$$
f: C P^{2 l-2} \rightarrow S U(2 l) / U(2 l-2),
$$

because $S^{4 l-3}$ is $4 l-4$ connected. Define $f_{+}$to be the basepoint preserving map induced by $f$ :

$$
f_{+}: C P_{+}^{2 l-2} \rightarrow S U(2 l) / U(2 l-2)+.
$$

Let $J: S U(2) \times S U(2 l) \rightarrow S U(2 l)$ be defined by $J(\alpha, \beta)=\beta \alpha \beta^{-1}$. As before $J$ descends to a map

$$
J: \Sigma^{3}\left(S U(2 l) / U(2 l-2)_{+}\right) \rightarrow S U(2 l) .
$$

(As previously mentioned, $J$ may be viewed as the basepointed adjoint of the inclusion $M(1, S U(2 l)) \rightarrow \Omega_{1}^{3} S U(2 l)$.) Boyer, Mann and Waggoner ([BMW], proof of Lemma 5.11) show that the composition

$$
\Sigma^{3}\left(C P_{+}^{2 l-2}\right) \stackrel{\Sigma^{3}\left(f_{+}\right)}{\longrightarrow} \Sigma^{3}\left(S U(2 l) / U(2 l-2)_{+}\right) \stackrel{J}{\longrightarrow} S U(2 l) \stackrel{p}{\rightarrow} S^{4 l-1},
$$

induces a surjection in $Z_{2}$-homology.

Now define an inclusion $\chi: S p(l) \hookrightarrow S U(2 l)$ as follows: given an $l \times l$ symplectic matrix $\left(x_{r s}\right)$, write

$$
x_{r s}=a_{r s}+i b_{r s}+j c_{r s}+k d_{r s}=a_{r s}+i b_{r s}+j\left(c_{r s}-i d_{r s}\right) .
$$

Form the $2 l \times 2 l$ complex matrix $\chi\left(\left(x_{r s}\right)\right)$ by replacing each entry $x_{r s}$ with the $2 \times 2$ matrix

$$
\left(\begin{array}{cc}
a_{r s}+i b_{r s} & -c_{r s}-i d_{r s} \\
c_{r s}-i d_{r s} & a_{r s}-i b_{r s}
\end{array}\right) .
$$

This corresponds to the natural inclusion of $S p(l) \hookrightarrow S U(2 l)$ if the skew form on $C^{2 l}$ is given by a block diagonal matrix where each block is the $2 \times 2$ matrix

$$
\left(\begin{array}{cc}
0 & -1 \\
1 & 0
\end{array}\right) \text {. }
$$

Notice that $\chi$ is defined so that the centralizer of $S p(1)$ in $S p(l)$ is mapped to the centralizer of $S U(2)$ in $S U(2 l)$. This allows us to define the map $\psi$, induced from $\chi$,

$$
\psi: S p(l) /\left(Z_{2} \times S p(l-1)\right) \rightarrow S U(2 l) / U(2 l-2) .
$$

Now consider the diagram

$$
\begin{aligned}
& \Sigma^{3}\left(R P_{+}^{4 l-1}\right)=\Sigma^{3}\left(S p(l) /\left(Z_{2} \times S p(l-1)\right)_{+}\right) \stackrel{\text { ad }\left(i_{1, l}\right)}{\longrightarrow} S p(l) \rightarrow S^{4 l-1} \\
& \Sigma^{3}\left(\psi_{+}\right) \downarrow \\
& \downarrow \chi \quad \downarrow=\text {. } \\
& \Sigma^{3}\left(S U(2 l) / U(2 l-2)_{+}\right) \quad \stackrel{J}{\longrightarrow} S U(2 l) \rightarrow S^{4 l-1}
\end{aligned}
$$


Because $\operatorname{ad}\left(i_{1, l}\right)$ and $J$ are defined in terms of conjugation, this diagram commutes. Thus, to show that the top composition induces a surjection it suffices to prove that $\left(\Sigma^{3}\left(\psi_{+}\right)\right)_{*}^{Z_{2}}$ is a surjection in dimension $4 l-1$. Equivalently, we must show that

$$
(\psi)_{*}^{Z_{2}}: H_{4 l-4}\left(S p(l) /\left(Z_{2} \times S p(l-1)\right) ; Z_{2}\right) \rightarrow H_{4 l-4}\left(S U(2 l) / U(2 l-2) ; Z_{2}\right)
$$

is a surjection. Note that there are isomorphisms

$$
H_{4 l-4}\left(S U(2 l) / U(2 l-2) ; Z_{2}\right) \cong H_{4 l-4}\left(M(1, S U(2 l)) ; Z_{2}\right) \cong H_{4 l-4}\left(C P^{2 l-1} ; Z_{2}\right) \cong Z_{2} .
$$

In fact there is a fibration

$$
U(2 l-1) / U(2 l-2) \longrightarrow S U(2 l) / U(2 l-2) \stackrel{\pi}{\longrightarrow} S U(2 l) / U(2 l-1)
$$

(which may be identified with the fibration $S^{4 l-3} \rightarrow M(1, S U(2 l)) \rightarrow C P^{2 l-1}$ as given in Section 4), where $U(2 l-1) \subset S U(2 l)$ consists of matrices of the form

$$
\left(\begin{array}{cc}
\operatorname{det}(B)^{-1} & 0 \\
0 & B
\end{array}\right)
$$

for $B \in U(2 n-1)$. Using the fact that the fiber is $4 l-4$-connected, we see from the Serre spectral sequence of this fibration that $(\pi)_{*}^{Z_{2}}$ is an isomorphism through dimension $4 l-4$. Hence, $(\psi)_{*}^{Z_{2}}$ is a surjection in dimension $4 l-4$ if $(\pi \circ \psi)_{*}^{Z_{2}}$ is. Because the composition $\pi \circ \psi$ may be viewed as a map from $R P^{4 l-1}$ to $C P^{2 l-1}$, the cohomology map $(\pi \circ \psi)_{Z_{2}}^{*}$ is an injection in dimension $4 n-4$ if and only if it is injective in dimension 2 . Since $H^{*} \cong \operatorname{Hom}\left(H_{*} ; Z_{2}\right)$, we see that $(\pi \circ \psi)$ will be a surjection in dimension $4 n-4$ if and only if it is a surjection in dimension 2. To finish the lemma, we examine the diagram

$$
\begin{aligned}
& R P^{3} \cong S p(1) / Z_{2} \stackrel{\rho_{1}}{\longrightarrow} S p(l) /\left(Z_{2} \times S p(l-1)\right) \cong R P^{4 l-1} \\
& \downarrow \cong \quad \downarrow \psi \\
& S U(2) / Z_{2} \quad \longrightarrow \quad S U(2 l) / U(2 l-2) \simeq M(1, S U(2 l)) \\
& \downarrow \downarrow \pi \\
& C P^{1} \cong S U(2) / S^{1} \stackrel{\rho_{2}}{\longrightarrow} S U(2 l) / U(2 l-1) \cong C P^{2 l-1},
\end{aligned}
$$

where the horizontal maps are induced by the inclusions of $S p(1) \hookrightarrow S p(l)$ and $S U(2) \hookrightarrow S U(2 l)$ defined earlier. Notice that the identification $U(2 l-1) \subset$ $S U(2 l)$ also stipulates the inclusion $S^{1} \hookrightarrow S U(2)$ to be

$$
\alpha \mapsto\left(\begin{array}{cc}
\alpha^{-1} & 0 \\
0 & \alpha
\end{array}\right)
$$

We leave it to the reader to check that these inclusions along with our identification $\chi: S p(l) \hookrightarrow S U(2 l)$, which induces $\psi$, make all of the maps in the diagram well-defined, and, therefore, that the diagram commutes. In addition, we see that $\rho_{1}$ is the inclusion of the three-skeleton $R P^{3} \hookrightarrow R P^{4 l-1}$, and $\rho_{2}$ is the inclusion of the two-skeleton $C P^{1} \hookrightarrow C P^{2 l-1}$, and so $\left(\rho_{1}\right)_{*}$ and $\left(\rho_{2}\right)_{*}$ 
are isomorphisms in dimension 2. Finally we remark that the composition of the two left vertical maps is just the natural projection

$$
R P^{3} \cong S^{3} / Z_{2} \longrightarrow S^{3} / S^{1} \cong C P^{1}
$$

which induces a $Z_{2}$-homology isomorphism in dimension 2 . Therefore, by the commutativity of the diagram, $(\pi \circ \psi)_{*}$ must be an isomorphism in dimension 2. This completes the proof of the lemma.

For the remainder of the discussion, all homology groups will be taken with $Z_{2}$ coefficients.

Lemma 17. The map $\Omega^{3} \operatorname{ad}\left(i_{1, l}\right): \Omega^{3} \Sigma^{3}\left(R P_{+}^{4 l-1}\right) \rightarrow \Omega^{3} S p(l)$ induces a surjection in homology with $Z_{2}$ coefficients.

Proof. Our proof proceeds by induction. For $l=1$ we are considering the $J$-homomorphism $\Omega^{3} \Sigma^{3}\left(R P_{+}^{3}\right) \rightarrow \Omega^{3} S^{3}$, which is known to induce a surjection in $Z_{2}$-homology. Now assume that $H_{*}\left(\Omega^{3} \operatorname{ad}\left(i_{1, l-1}\right)\right)$ is a surjection. Define $R P_{4 l-4}^{4 l-1}$ to be the stunted projective space

$$
R P_{4 l-4}^{4 l-1}=R P_{+}^{4 l-1} / R P_{+}^{4 l-5}
$$

Let $p_{l}$ be the projection $p_{l}: R P_{+}^{4 l-1} \rightarrow R P_{4 l-4}^{4 l-1}$, and let $\mathscr{F}$ denote the homotopy fiber of

$$
\Sigma^{3} p_{l}: \Sigma^{3}\left(R P_{+}^{4 l-1}\right) \rightarrow \Sigma^{3} R P_{4 l-4}^{4 l-1}
$$

Since the diagram

$$
\begin{array}{ccc}
\Sigma^{3} R P_{+}^{4 l-5} & \stackrel{\operatorname{ad}\left(i_{1, l-1}\right)}{\longrightarrow} & S p(l-1) \\
\downarrow & \downarrow \\
\Sigma^{3} R P_{+}^{4 l-1} & \stackrel{\operatorname{ad}\left(i_{1, l}\right)}{\longrightarrow} & S p(l)
\end{array}
$$

commutes, we obtain a map

$$
\eta_{l}: \Sigma^{3} R P_{4 l-4}^{4 l-1} \longrightarrow S^{4 l-1}=\Sigma^{3} S^{4 l-4}
$$

which, by the previous lemma, must induce a surjection in $Z_{2}$-homology. In particular, if we let $\theta \in H_{4 l-4}\left(S^{4 l-4}\right)$ be the generator, then $\Sigma^{3} \theta$ is in the image of $H_{*}\left(\eta_{l}\right)$. Notice that by the connectivity of $S^{4 l-4}$, there exists a map $f: R P_{4 l-4}^{4 l-1} \rightarrow S^{4 l-4}$ such that $\eta \simeq \Sigma^{3} f$. (See, for example, [H].) Thus, if $\Sigma^{3} \theta$ is in the image of $H_{*}(\eta)$, then $\theta$ is in the image of $H_{*}(f)$. We will soon show that this forces $\Omega^{3} \eta_{l}$ to be a surjection. 
Applying $\Omega^{3}$ we get maps

$$
\begin{aligned}
& \left.\left.\Omega^{3} \Sigma^{3} R P_{+}^{4 l-5} \stackrel{\Omega^{3}\left(\operatorname { a d } \left(i_{1}, l-1\right.\right.}{\longrightarrow}\right)\right) \quad \Omega^{3} S p(l-1) \\
& \Omega^{3} \Sigma^{3} R P_{+}^{4 l-1} \quad \stackrel{\Omega^{3}\left(\operatorname{ad}\left(i_{1}, l\right)\right)}{\longrightarrow} \quad \Omega^{3} S p(l) \\
& \downarrow \\
& \Omega^{3} \Sigma^{3} R P_{4 l-4}^{4 l-1} \quad \stackrel{\Omega^{3} \eta_{l}}{\longrightarrow} \quad \Omega^{3} \Sigma^{3} S^{4 l-4},
\end{aligned}
$$

where the right-hand column is the three-fold looping of the fibration

$$
S p(l-1) \hookrightarrow S p(l) \stackrel{\text { proj }}{\longrightarrow} S^{4 l-1} .
$$

We can replace $\Omega^{3} \Sigma^{3} R P_{+}^{4 l-5}$ with $\Omega^{3} \mathscr{F}$ to obtain the following map of fibrations (in the columns):

$$
\begin{array}{ccccc}
\Omega^{3} \Sigma^{3} R P_{+}^{4 l-5} & \longrightarrow & \Omega^{3} \mathscr{F} & \longrightarrow & \Omega^{3} S p(l-1) \\
\downarrow & \downarrow & & \downarrow \\
\Omega^{3} \Sigma^{3} R P_{+}^{4 l-5} & \longrightarrow & \Omega^{3} \Sigma^{3} R P_{+}^{4 l-1} & \longrightarrow & \Omega^{3} S p(l) \\
\downarrow & & \downarrow & & \downarrow \\
\{+\} & \longrightarrow & \Omega^{3} \Sigma^{3} R P_{4 l-4}^{4 l-1} & \longrightarrow & \Omega^{3} \Sigma^{3} S^{4 l-4},
\end{array}
$$

where the map $\Omega^{3} \mathscr{F} \rightarrow \Omega^{3} S p(l-1)$ is any lifting up to homotopy of the composition $\Omega^{3} \mathscr{F} \rightarrow \Omega^{3} \Sigma^{3}\left(R P_{+}^{4 l-1}\right) \rightarrow \Omega^{3} S p(l)$. Comparing $H_{*}\left(\Omega^{3} \Sigma^{3}\left(R P_{+}^{4 l-1}\right)\right)$ with $H_{*}\left(\Omega^{3} \Sigma^{3}\left(R P_{4 l-4}^{4 l-1}\right)\right)$ using results of F. Cohen ([CLM] III, Lemma 3.8), we can show that the map $\Omega^{3} \Sigma^{3} p_{l}: \Omega^{3} \Sigma^{3}\left(R P_{+}^{4 l-1}\right) \rightarrow \Omega^{3} \Sigma^{3} R P_{4 l-4}^{4 l-1}$ induces a surjection in $Z_{2}$-homology. To see this, let $X$ denote any sufficiently nice space, for example, a finite C.W. complex, let $i \in\{1,2\}$, let $Q_{i}: H_{q}\left(\Omega^{3} \Sigma^{3} X\right) \rightarrow$ $H_{2 q+i}\left(\Omega^{3} \Sigma^{3} X\right)$ be a Dyer-Lashof operation, and let $\lambda_{2}: H_{q}\left(\Omega^{3} \Sigma^{3} X\right) \otimes$ $H_{r}\left(\Omega^{3} \Sigma^{3} X\right) \rightarrow H_{q+r+2}\left(\Omega^{3} \Sigma^{3} X\right)$ be the (possibly) nontrivial Browder operation. Let $I=\left\{i_{1} \leq i_{2} \leq \cdots \leq i_{r}\right\}$ be a nondecreasing sequence, where $i_{j} \in\{1,2\}$, and $Q_{I}$ denote the iterated Dyer-Lashof operation $Q_{I}=Q_{i_{1}} Q_{i_{2}} \cdots Q_{i_{r}}$. It follows from work of F. Cohen [CLM] that $H_{*}\left(\Omega^{3} \Sigma^{3} X\right)$ is, essentially, the commutative algebra generated by elements of the form $Q_{I}(y)$ where $y$ is an iterated Browder operation on elements of $H_{*}(X)$. (As written, this set of generators does not generate $H_{*}\left(\Omega^{3} \Sigma^{3} X\right)$ freely, but it does generate it.) Now suppose that $y$ is an iterated Browder operation on elements $\gamma_{1}, \ldots, \gamma_{s} \in H_{*}\left(R P_{4 l-4}^{4 l-1}\right)$. We write $y \in \lambda_{2}\left(\left\{\gamma_{1}, \ldots, \gamma_{s}\right\}\right)$ to denote the fact that $y$ may be written using only the symbols $\lambda_{2}, \gamma_{1}, \ldots, \gamma_{s}$. For example, if $y=\lambda_{2}\left[\lambda_{2}\left[\gamma_{1}, \gamma_{3}\right], \gamma_{2}\right]$, then $y \in \lambda_{2}\left(\left\{\gamma_{1}, \gamma_{2}, \gamma_{3}\right\}\right)$. There is a natural inclusion $H_{*}\left(R P_{4 l-4}^{4 l-1}\right) \hookrightarrow H_{*}\left(R P_{+}^{4 l-1}\right)$ 
which sends $\gamma_{i}$ to, say, $\tilde{\gamma}_{i} \in H_{*}\left(R P_{+}^{4 l-1}\right)$, and the natural projection $p_{l}$ induces a map sending $\tilde{\gamma}_{i}$ to $\gamma_{i}$. Since the map

$$
\Omega^{3} \Sigma^{3} p_{l}: \Omega^{3} \Sigma^{3}\left(R P_{+}^{4 l-1}\right) \rightarrow \Omega^{3} \Sigma^{3} R P_{4 l-4}^{4 l-1}
$$

respects the Browder operations, there exists a $\tilde{y} \in H_{*}\left(\Omega^{3} \Sigma^{3}\left(R P_{+}^{4 l-1}\right)\right)$ such that $\tilde{y} \in \lambda_{2}\left(\left\{\tilde{\gamma}_{1}, \ldots, \tilde{\gamma}_{s}\right\}\right)$ and $\tilde{y} \mapsto y$ under the induced map $H_{*}\left(\Omega^{3} \Sigma^{3} p_{l}\right)$. Moreover, for any sequence $I$, the naturality of the Dyer-Lashof operations with respect to iterated loop maps shows that $Q_{I}(\tilde{y})$ maps to $Q_{I}(y)$. Thus, a set of algebra generators for $H_{*}\left(\Omega^{3} \Sigma^{3} R P_{4 l-4}^{4 l-1}\right)$ is in the image of $H_{*}\left(\Omega^{3} \Sigma^{3} p_{l}\right)$. Since this map preserves products, it must be a surjection.

By similar reasoning we see that the map

$$
\Omega^{3} \eta_{l}: \Omega^{3} \Sigma^{3} R P_{4 l-4}^{4 l-1} \rightarrow \Omega^{3} \Sigma^{3} S^{4 l-4}
$$

induces a surjection in $Z_{2}$ homology: recall that there is a map $f: R P_{4 l-4}^{4 l-1} \rightarrow$ $S^{4 l-4}$ such that $\Sigma^{3} f \simeq \eta_{l}$, and that by a previous lemma there is a $\tilde{\theta} \in$ $H_{*}\left(R P_{4 l-4}^{41-1}\right)$ such that $H_{*}(f)(\tilde{\theta})$ is the generator $\theta \in H_{4 l-4}\left(S^{4 l-4}\right)$. Therefore, for any iterated Dyer-Lashof operation $Q_{I}$, we have

$$
H_{*}\left(\Omega^{3} \eta_{l}\right)\left(Q_{I}(\tilde{\theta})\right)=H_{*}\left(\Omega^{3} \Sigma^{3} f\right)\left(Q_{I}(\tilde{\theta})\right)=Q_{I}(\theta) .
$$

Since $H_{*}\left(\Omega^{3} \Sigma^{3} S^{4 l-4}\right)$ is generated by such $Q_{I}(\theta), H_{*}\left(\Omega^{3} \eta_{l}\right)$ is a surjection.

Because $\Omega^{3} \Sigma^{3} p_{l}$ and $\Omega^{3} \eta_{l}$ induce surjections and the diagram

$$
\begin{array}{lcc}
\Omega^{3} \Sigma^{3}\left(R P_{+}^{4 l-1}\right) & \longrightarrow & \Omega^{3} S p(l) \\
\Omega^{3} \Sigma^{3} p_{l} \downarrow & \downarrow \\
\Omega^{3} \Sigma^{3} R P_{4 l-4}^{4 l-1} & \stackrel{\Omega^{3} \eta_{l}}{\longrightarrow} & \Omega^{3} \Sigma^{3} S^{4 l-4},
\end{array}
$$

commutes, the map $\Omega^{3} S p(l) \rightarrow \Omega^{3} \Sigma^{3} S^{4 l-4}$ must also induce a surjection in $Z_{2}$ homology. We can now conclude that the Serre spectral sequences of all three fibrations in the earlier diagram collapse at the $E_{2}$ level. This is a consequence of the following general fact.

Proposition 18. Let $F \rightarrow E \stackrel{p}{\rightarrow} B$ be a principal fibration, $\pi_{i}(B)=0$ for $i \leq 2$. Suppose the natural map $\Omega p: \Omega E \rightarrow \Omega B$ induces a surjection in $Z_{2}$-homology. Then the Serre spectral sequence for the fibration $\Omega F \rightarrow \Omega E \rightarrow \Omega B$ collapses at $E_{2}$.

Proof. First note that $E_{2}=H_{*}(\Omega B) \otimes \mathrm{H}_{*}(\Omega F)$. Also note that $\Omega F$ is an $H$ space and, therefore, $H_{*}(\Omega F)$ is an algebra. Consequently, $H_{*}(\Omega F)$ acts on $E_{2}$. In fact, since the fibration $\Omega F \rightarrow \Omega E \rightarrow \Omega B$ is principal, the differentials $d_{r}$ act as derivations with respect to the natural induced action of $H_{*}(\Omega F)$ on $E_{r}$ [MT]. Now let $\alpha \otimes \beta \in H_{*}(\Omega B) \otimes H_{*}(\Omega F)$ be an element of $E_{2}$. Then

$$
d_{2}(\alpha \otimes \beta)=d_{2}((\alpha \otimes 1) \cdot \beta)=d_{2}(\alpha \otimes 1) \cdot \beta=0,
$$

the last equality holding because $H_{*}(\Omega p)$ is a surjection and hence $\alpha \otimes 1$ must survive to $E_{\infty}$. Thus $E_{2} \cong E_{3}$. We can repeat this argument on the $E_{r}$ term 
using differential $d_{r}$ to conclude that that $E_{3} \cong E_{4} \cong \ldots \cong E_{\infty}$. This proves the proposition.

Recall that our goal is to show that the map

$$
\Omega^{3}\left(\operatorname{ad}\left(i_{1, l}\right)\right): \Omega^{3} \Sigma^{3}\left(R P_{+}^{4 l-1}\right) \rightarrow \Omega^{3} S p(l)
$$

induces a surjection in $Z_{2}$-homology, and we may inductively assume that the map $H_{*}\left(\Omega^{3}\left(\operatorname{ad}\left(i_{1, l-1}\right)\right)\right)$ is a surjection. Now from the collapsing of the spectral sequences we have that $\Omega^{3} S p(l-1) \rightarrow \Omega^{3} S p(l)$ induces an injection in $Z_{2}$ homology. Looking back at the diagram of the three fibrations and using our inductive assumption, we can conclude that the composition

$$
\Omega^{3} \Sigma^{3}\left(R P_{+}^{4 l-5}\right) \rightarrow \Omega^{3} \mathscr{F} \rightarrow \Omega^{3} S p(l-1)
$$

also induces a surjection in $Z_{2}$-homology. To see this, recall the commutative diagram

$$
\begin{aligned}
& \Omega^{3} \Sigma^{3}\left(R P_{+}^{4 l-5}\right) \stackrel{\Omega^{3}\left(\mathbf{a d}\left(i_{1, l-1}\right)\right)}{\longrightarrow} \quad \Omega^{3} S p(l-1) \\
& \downarrow \\
& \Omega^{3} \Sigma^{3}\left(R P_{+}^{4 l-1}\right) \quad \stackrel{\Omega^{3}}{\left.\stackrel{\operatorname{ad}\left(i_{1}, l\right.}{\longrightarrow}\right)} \quad \Omega^{3} S p(l),
\end{aligned}
$$

and let $d_{i}=\operatorname{dim}\left(H_{i}\left(\Omega^{3} S p(l-1)\right)\right.$. By our inductive assumption and the fact that

$$
H_{*}\left(\Omega^{3} S p(l-1)\right) \rightarrow H_{*}\left(\Omega^{3} S p(l)\right)
$$

is an injection, the dimension of the image of the composition

$$
H_{i}\left(\Omega^{3} \Sigma^{3}\left(R P_{+}^{4 l-5}\right)\right) \rightarrow H_{i}\left(\Omega^{3} \Sigma^{3}\left(R P_{+}^{4 l-1}\right)\right) \rightarrow H_{i}\left(\Omega^{3} S p(l)\right)
$$

must be $d_{i}$. By the homotopy commutativity of

$$
\begin{array}{ccccc}
\Omega^{3} \Sigma^{3}\left(R P_{+}^{4 l-5}\right) & \rightarrow & \Omega^{3} \mathscr{F} & \rightarrow & \Omega^{3} S p(l-1) \\
=\downarrow & \downarrow & & \downarrow \\
\Omega^{3} \Sigma^{3}\left(R P_{+}^{4 l-5}\right) & \rightarrow & \Omega^{3} \Sigma^{3}\left(R P_{+}^{4 l-1}\right) & \rightarrow & \Omega^{3} S p(l),
\end{array}
$$

the dimension of the composition

$$
H_{i}\left(\Omega^{3} \Sigma^{3}\left(R P_{+}^{4 l-5}\right)\right) \rightarrow H_{i}\left(\Omega^{3} \mathscr{F}\right) \rightarrow H_{i}\left(\Omega^{3} S p(l-1)\right) \rightarrow H_{i}\left(\Omega^{3} S p(l)\right)
$$

must also be $d_{i}$. Hence, the composition

$$
H_{*}\left(\Omega^{3} \Sigma^{3}\left(R P_{+}^{4 l-5}\right)\right) \rightarrow H_{i}\left(\Omega^{3} \mathscr{F}\right) \rightarrow H_{i}\left(\Omega^{3} S p(l-1)\right)
$$

is a surjection. 
To summarize what we have shown thus far, we have the map of fibrations

$$
\begin{array}{ccc}
\Omega^{3} \mathscr{F} & \longrightarrow & \Omega^{3} S p(l-1) \\
\downarrow & \downarrow \\
\Omega^{3} \Sigma^{3}\left(R P_{+}^{4 l-1}\right) & \stackrel{\Omega^{3}\left(\operatorname{ad}\left(i_{1}, l\right)\right)}{\longrightarrow} & \Omega^{3} S p(l) \\
\downarrow & & \downarrow \\
\Omega^{3} \Sigma^{3} R P_{4 l-4}^{4 l-1} & \stackrel{\Omega^{3} \eta_{l}}{\longrightarrow} & \Omega^{3} \Sigma^{3} S^{4 l-4},
\end{array}
$$

where the induced maps $H_{*}\left(\Omega^{3} \mathscr{F}\right) \rightarrow H_{*}\left(\Omega^{3} S p(l-1)\right)$ and $H_{*}\left(\Omega^{3} \eta_{l}\right)$ are surjections. In other words, the $E_{2}$ term of the Serre spectral sequence of the left fibration surjects onto the $E_{2}$ term of the right fibration. But we also have shown that both spectral sequences collapse at $E_{2}$. Hence, the $E_{\infty}$ term of the left fibration surjects onto the $E_{\infty}$ term of the right fibration. Now, by the convergence of the spectral sequences, a standard argument on the level of filtrations shows that the map of fibrations $\Omega^{3}\left(\operatorname{ad}\left(i_{1, l}\right)\right)$ induces a surjection in $Z_{2}$-homology. This finishes the proof of Lemma 17.

For future use, we record as a corollary one of the facts demonstrated during the proof of the lemma.

Corollary 19. The natural map into the limit space $\Omega^{3} S p(l) \rightarrow Z \times B O$ induces an injection in homology with $Z_{2}$ coefficients.

Proof. We have shown that for any $l$ the map $H_{*}\left(\Omega^{3} S p(l)\right) \rightarrow H_{*}\left(\Omega^{3} S p(l+1)\right)$ is an injection. Hence, $H_{*}\left(\Omega^{3} S p(l)\right)$ injects into $H_{*}\left(\Omega^{3} S p\right) \cong H_{*}(Z \times B O)$.

We now return to the proof of the theorem. From the lemma, we know that the composite

$$
H_{*}\left(\Omega^{3} \Sigma^{3} R P_{+}^{4 l-1}\right) \longrightarrow H_{*}\left(\Omega^{3} S p(l)\right) \longrightarrow H_{*}(Z \times B O)
$$

has the same image as the image of $H_{*}\left(\Omega^{3} S p(l)\right)$. This image is easily calculated as follows: let $b_{i} \in H_{*}(B O(1))$ be a generator. Then $H_{*}(Z \times B O) \cong$ $Z_{2}\left[b_{0}, b_{0}^{-1}, b_{1}, b_{2}, \ldots\right]$. By the commutativity of

$$
\begin{array}{cccc}
R P^{4 l-1} \stackrel{h}{\longrightarrow} & M(1, S p(l)) & \stackrel{\partial_{S p}}{\longrightarrow} & B O(1) \\
& \downarrow & & \downarrow \\
& \Omega_{1}^{3} S p(l) & \stackrel{\beta_{S p(l), 1}}{\longrightarrow} & \{1\} \times B O,
\end{array}
$$

the fact that $\partial_{S p} \circ h$ is homotopic to the natural inclusion $R P^{4 l-1} \rightarrow R P^{\infty}$, and what we know about the homology of $\Omega^{3} \Sigma^{3} R P_{+}^{4 l-1}$ in terms of the homology of $R P^{4 l-1}$ [CLM], we see that the image $\operatorname{Im}\left(H_{*}\left(\Omega^{3} \Sigma^{3} R P_{+}^{4 l-1}\right)\right) \subset H_{*}(Z \times B O)$ consists of linear combinations of products of certain iterated Dyer-Lashof operations on $b_{0}, b_{0}^{-1}, b_{1}, \ldots, b_{4 l-1}$. (There are no Browder operations in the image because $Z \times B O$ is an infinite loop space.) 
Now we are ready to calculate the intersection of the maps

$$
\begin{gathered}
H_{*}\left(\coprod_{0 \leq j \leq k} B O(j)\right) \\
\downarrow \\
H_{*}\left(\Omega^{3} S p(l)\right) \longrightarrow \quad H_{*}(Z \times B O) .
\end{gathered}
$$

and prove the theorem. Just as in unitary case, the $\operatorname{Im}\left(\coprod_{0 \leq j \leq k} H_{*}(B O(j))\right)$ $\subset H_{*}(Z \times B O)$ is all polynomials in $b_{0}, b_{1}, \ldots$, such that each term has length $\leq k$. Suppose $\alpha$ is such a homology class, and further suppose that $\alpha$ is in the image of $H_{*}\left(\Omega^{3} S p(l)\right)$. By the previous lemma there exists $\tilde{\alpha} \in$ $H_{*}\left(\Omega^{3} \Sigma^{3} R P_{+}^{4 l-1}\right)$ which maps to $\alpha$ under the composition

$$
H_{*}\left(\Omega^{3} \Sigma^{3} R P_{\downarrow^{4 l-1}}^{4 l}\right) \longrightarrow H_{*}\left(\Omega^{3} S p(l)\right) \longrightarrow H_{*}(Z \times B O) .
$$

Since $\tilde{\alpha}$ can be chosen to be a linear combination of products of iterated DyerLashof operations $Q_{1}, Q_{2}$, and our maps are three-fold loop maps respecting the Dyer-Lashof operations, $\alpha$ can be expressed as a sum of terms of the form

$$
Q_{I_{1}}\left(b_{j_{1}}\right) Q_{I_{2}}\left(b_{j_{2}}\right) \cdots Q_{I_{n}}\left(b_{j_{n}}\right), \quad j_{r} \geq 0 .
$$

By calculations of Kochman,[Ko], each such term is a polynomial in $b_{0}, b_{1}, \ldots$ which has length

$$
2^{L\left(I_{1}\right)}+2^{L\left(I_{2}\right)}+\cdots+2^{L\left(I_{n}\right)}
$$

where $L\left(I_{r}\right)$ is the number of elements of the sequence $I_{r}$. Since $\alpha$ is in the image of $H_{*}\left(\coprod_{0 \leq j \leq k} B O(j)\right)$, we must have

$$
\sum_{r=1}^{n} 2^{L\left(I_{r}\right)} \leq k .
$$

Let $t(\tilde{\alpha})=Q_{I_{1}}\left(b_{j_{1}}\right) \cdots Q_{I_{n}}\left(b_{j_{n}}\right) \in H_{*}\left(\Omega^{3} \Sigma^{3} R P_{+}^{4 l-1}\right)$ be one term of some expression for $\tilde{\alpha} . t(\tilde{\alpha})$ maps to the element $Q_{I_{1}}\left(b_{j_{1}}\right) \cdots Q_{I_{n}}\left(b_{j_{n}}\right) \in H_{*}(Z \times B O)$. Notice that in F. Cohen's [CLM] terminology, the weight of $t(\tilde{\alpha})$ equals $\sum_{r} 2^{L\left(I_{r}\right)}$ which is $\leq k$. By another result of F. Cohen [CLM], this implies that $t(\tilde{\alpha})$ is in the image of the natural map

$$
H_{*}\left(F_{k}\left(R P_{+}^{4 l-1}\right)\right) \longrightarrow H_{*}\left(\Omega^{3} \Sigma^{3}\left(R P_{+}^{4 l-1}\right)\right) .
$$

In summary we have the following picture:

$$
\begin{aligned}
& H_{*}\left(F_{k}\left(R P_{+}^{4 l-1}\right)\right) \quad \stackrel{\cong}{\longrightarrow} H_{*}\left(F_{k}\left(M(1, S p(l))_{+}\right)\right) \quad \longrightarrow \quad H_{*}\left(F_{k}\left(R P_{+}^{\infty}\right)\right) \\
& \text { (i) } \\
& \downarrow \\
& \downarrow \quad H_{*}\left(\amalg_{0 \leq j \leq k} M(j, S p(l))\right) \stackrel{\amalg_{j}^{\left(\partial_{S p}\right) *}}{\longrightarrow} H_{*}\left(\amalg_{0 \leq j \leq k} B O(j)\right) \\
& H_{*}\left(\Omega^{3} \Sigma^{3}\left(R P_{+}^{4 l-1}\right)\right) \stackrel{\left(\Omega^{3}\left(a d\left(i_{1}, l\right)\right)\right) *}{\longrightarrow} \quad H_{*}\left(\Omega^{3} S p(l)\right) \quad \stackrel{\left(\beta_{S p(l)}\right) *}{\longrightarrow} \quad H_{*}(Z \times B O)
\end{aligned}
$$


where we know that (ii) and (iii) commute. (i) will commute because the outer most square

$$
\begin{array}{ccc}
H_{*}\left(F_{k}\left(R P_{+}^{4 l-1}\right)\right) & \longrightarrow & H_{*}\left(F_{k}\left(R P_{+}^{\infty}\right)\right) \\
\downarrow & \downarrow \\
H_{*}\left(\Omega^{3} \Sigma^{3} R P_{+}^{4 l-1}\right) & \longrightarrow & H_{*}(Z \times B O)
\end{array}
$$

commutes and the map

$$
H_{*}\left(\Omega^{3} S p(l)\right) \longrightarrow H_{*}(Z \times B O)
$$

is an injection. Furthermore, we have already established that for each $\alpha \in$ $H_{*}(Z \times B O)$ which is in the image of $H_{*}\left(\coprod_{0 \leq j \leq k} B O(j)\right)$ and the image of $H_{*}\left(\Omega^{3} S p(l)\right)$, there is a $\gamma \in H_{*}\left(F_{k}\left(R P_{+}^{4 l-1}\right)\right)$ which maps to $\alpha$ under either composition in the above square. Thus, by the commutativity of (i), the image of $\gamma$ under the homology gluing map

$$
H_{*}\left(F_{k}\left(R P_{+}^{4 l-1}\right)\right) \rightarrow H_{*}\left(\coprod_{0 \leq j \leq k} M(j, S p(l))\right)
$$

must map to $\alpha$. This completes the proof of the theorem.

\section{REFERENCES}

[A] M. F. Atiyah, Bott periodicity and the index of elliptic operators, Quart. J. Math. Oxford 19 (1968), 113-140.

[AHS] M.F. Atiyah, N.J. Hitchen, and I.M. Singer, Self-duality in four dimensional Riemannian geometry, Proc. Roy. Soc. London A 362 (1978), 425-461.

[ADHM] M.F. Atiyah, V. G. Drinfeld, N.J. Hitchin, and Y. I. Manin, Construction of instantons, Phys. Lett. A 65 (1978), 185-187.

[AJ] M.F. Atiyah and J.D.S. Jones, Topological aspects of Yang-Mills theory, Comm. Math. Phys. 61 (1978), 97-118.

[BHMM] C.P. Boyer, J.C. Hurtubise, B.M. Mann, and R.J. Milgram, The topology of instanton moduli spaces I: The Atiyah-Jones conjecture, Ann. of Math. 137 (1993), 561-609.

[BM] C.P. Boyer and B.M. Mann, Homology operations on instantons, J. Differential Geom. 28 (1988), 423-465.

[BMW] C.P. Boyer, B.M. Mann, and D. Waggoner, On the homology of SU(n) instantons, Trans. Amer. Math. Soc. 323 (1991), 529-561.

[CJ] R. Cohen and J.D.S. Jones, Monopoles, braid groups, and the Dirac operator, Comm. Math. Phys. 158 (1993), 241-246.

[CLM] F. R. Cohen, T. J. Lada, and J.P. May, The homology of iterated loop spaces, Lecture Notes in Math., vol. 533, Springer-Verlag, 1976.

[D] S.K. Donaldson, Instantons and geometric invariant theory, Comm. Math. Phys. 93 (1984), 453-460.

[DK] S. K. Donaldson and P.B. Kronheimer, The geometry of four-manifolds, Oxford Math. Monographs, Oxford Univ. Press, Oxford, 1990.

[H] D. Husemoller, Fibre bundles, Springer-Verlag, New York, 1966.

[K] F. Kirwan, Geometric invariant theory and the Atiyah-Jones conjecture, Proceedings of the Sophus Lie Memorial Conference (Oslo, 1992), editors, O. A. Laudal and B. Jahren, Scandinavian University Press, 1994, pp. 161-188.

[Ko] S. Kochman, The homology of the classical groups over the Dyer-Lashof algebra, Bull. Amer. Math. Soc. 77 (1971), 142-147. 
[Mu J J.R. Munkres, Elementary differential topology, Ann. of Math. 54, Princeton Univ. Press, Princeton, NJ, 1963.

[MT] M. Mimura and H. Toda, Topology of Lie groups, I and II, Transl. Math. Monographs, vol. 91, Amer. Math. Soc., Providence, RI, 1991.

[OSS] C. Okonek, M. Schneider, and H. Spindler, Vector bundles on complex projective spaces, Birkhäuser, Boston, 1980.

[P] S. Priddy, Dyer-Lashofoperations for the classifying spaces of certain matrix groups, Quart. J. Math. Oxford 26 (1975), 179-193.

[Ti] Y. Tian, The based SU(n)-instanton moduli spaces, Math. Ann. 298 (1994), 117-139.

[T1] C. H. Taubes, Self-dual connections on four-manifolds with indefinite intersection matrix, J. Differential Geom. 19 (1984), 517-560.

[T2] C. H. Taubes, The stable topology of selfdual moduli spaces, J. Differential Geom. 29 (1989), 163-230.

Department of Mathematics, University of Minnesota, Minneapolis, Minnesota 55455

E-mail address: sanders@math.umn. edu 